MATHEMATICS OF COMPUTATION

Volume 70, Number 233, Pages 135-172

S $0025-5718(00) 01201-1$

Article electronically published on March 2, 2000

\title{
WEAK APPROXIMATIONS. A MALLIAVIN CALCULUS APPROACH
}

\author{
ARTURO KOHATSU-HIGA
}

\begin{abstract}
We introduce a variation of the proof for weak approximations that is suitable for studying the densities of stochastic processes which are evaluations of the flow generated by a stochastic differential equation on a random variable that may be anticipating. Our main assumption is that the process and the initial random variable have to be smooth in the Malliavin sense. Furthermore, if the inverse of the Malliavin covariance matrix associated with the process under consideration is sufficiently integrable, then approximations for densities and distributions can also be achieved. We apply these ideas to the case of stochastic differential equations with boundary conditions and the composition of two diffusions.
\end{abstract}

\section{INTRODUCTION}

Starting as early as Milshtein [22, due to its many applications the area of weak approximations for stochastic differential equations (sde's) has been growing rapidly (e.g. see Kloeden and Platen [16]).

To explain what are the issues in this area of study, let's consider a simple case first. Define $\Phi_{t}(x)$ as the solution of the following one dimensional stochastic differential equation:

$$
\Phi_{t}(x)=x+\int_{0}^{t} \sigma\left(\Phi_{s}(x)\right) \circ d W_{s}+\int_{0}^{t} b\left(\Phi_{s}(u, x)\right) d s, \quad t \in[0,1] .
$$

Here $W$ is a one dimensional Wiener process and $b, \sigma: \mathbb{R} \rightarrow \mathbb{R}$ are smooth with bounded derivatives. The above stochastic integral is the Stratonovich integral.

The main problem of study in the area of strong and weak approximation theory is how to approximate $\Phi$ and what is the error of approximation. For this we define the Euler approximation. Let $\pi=\left\{0=t_{0}<\ldots<t_{N}=1\right\}$ be a partition of $[0,1]$ such that $\|\pi\|:=\max \left\{t_{k+1}-t_{k} ; k=0, \ldots, N-1\right\} \leq \delta$. Define the shift operator

$$
\eta(t)=t_{k} \quad \text { if } t_{k}<t \leq t_{k+1}
$$

Received by the editor June 9, 1998 and, in revised form March 2, 1999.

2000 Mathematics Subject Classification. Primary 60H07, 60H35, 65C30, 34B99.

Key words and phrases. Stochastic differential equations, boundary conditions, weak approximation, numerical analysis.

This article was partially written while the author was visiting the Department of Mathematics at Kyoto University with a JSPS fellowship. His research was partially supported by a DGES grant.

(C) 2000 by Arturo Kohatsu-Higa 
where $t_{k}$ and $t_{k+1}$ are in $\pi$. We set $\eta(0)=0$. Let $\bar{\Phi}$ denote the Euler-Maruyama scheme, defined by

$$
\bar{\Phi}_{t}(x)=x+\int_{0}^{t} \sigma\left(\bar{\Phi}_{\eta(s)}(x)\right) d W_{s}+\int_{0}^{t} m\left(\bar{\Phi}_{\eta(s)}(x)\right) d s .
$$

Here $m=b+\frac{1}{2} \sigma^{\prime} \sigma$.

A well known result (see e.g. Theorem 10.2.2 in [16]) establishes that

$$
E\left(\sup _{t \in[0,1]}\left|\Phi_{t}(x)-\bar{\Phi}_{t}(x)\right|^{p}\right) \leq C(x) \delta^{p / 2} .
$$

Here $p>1$, and $C(x)$ is a positive function that has polynomial growth in $x$ and is independent of $\delta$ and the partition $\pi$.

This type of result measures the path-by-path difference between the solution of (1) and its Euler-Maruyama approximation (2). For this reason this type of result is usually called a strong approximation theorem. Its method of proof is based on Gronwall's lemma.

A different way of measuring the difference between $\Phi$ and $\bar{\Phi}$ is through their laws. In particular the following result holds (see, e.g. Theorem 14.1.5 in [16]):

$$
\left|E\left(f\left(\Phi_{t}(x)\right)\right)-E\left(f\left(\bar{\Phi}_{t}(x)\right)\right)\right| \leq C(x) \delta,
$$

where $f \in C_{p}^{\infty}(\mathbb{R})$ (that is, $f$ is a real valued, smooth function with polynomial growth at infinity). This type of result is different from (3). In fact, if $f$ above were Lipschitz (with Lipschitz constant $K$ ), using (3) we could only obtain that

$$
\left|E\left(f\left(\Phi_{t}(x)\right)\right)-E\left(f\left(\bar{\Phi}_{t}(x)\right)\right)\right| \leq K E\left(\left|\Phi_{t}(x)-\bar{\Phi}_{t}(x)\right|\right) \leq K C(x) \delta^{1 / 2},
$$

while (4) establishes that the rate of convergence is of order $\delta$. Results like (4) are known as weak approximation theorems. The method of proof is centered on the fact that if we define $u(t, x)=E\left(f\left(\Phi_{t}(x)\right)\right)$, then $u$ satisfies the following PDE:

$$
\begin{aligned}
& \frac{\partial u}{\partial s}(s, x)+m(x) \frac{\partial u}{\partial x}(s, x)+\frac{1}{2} \sigma^{2}(x) \frac{\partial^{2} u}{\partial x^{2}}(s, x)=0, \\
& u(0, x)=f(x) .
\end{aligned}
$$

Having defined $u$, one rewrites (4) in terms of $u$ and applies Itô's formula. At some point during the proof the Markov property of the process $\Phi_{t}(x)$ is used.

Due to its connections with partial differential equations, the area of weak approximations has recently been of increasing interest. Also, results of this type give information about many functionals of the solution process, in particular, the moments and the law of the process $\Phi_{t}(x)$. For example, Bally and Talay [3], [4] widened the class where $f$ belongs to have a result like (4) hold. In particular, one can obtain approximations for the distribution and density functions of $\Phi$. Other related results were developed by $\mathrm{Hu}$ and Watanabe [14] and Kohatsu-Higa [17.

Another direction of development has been to consider other stochastic differential equations of a type different from (1). For example, Bossy and Talay [5] and [6] considered stochastic differential equations related to the Burgers and McKeanVlasov equations. These stochastic equations are of a type different from (1). In particular, the law of the solution process is also part of the coefficients in the equation.

In all these variants the essential technique used to obtain a result like (4) is to find an appropiate modification of the basic argument that uses $u$ (which also 
needs to be modified), the adaptedness and the Markov property of the underlying stochastic process.

Until recently, it was common to believe that anticipating stochastic differential equations were not amenable to this type of argument for studying the numerical approximations, due to the lack of adapted properties and the Markov property. In Ahn and Kohatsu-Higa 2, we defined and analyzed the weak and strong rate of convergence for an Euler type scheme in the case of $\Phi_{t}\left(X_{0}\right)$, where $X_{0}$ is a smooth random variable (in the Malliavin sense) but not necessarily adapted to $\mathcal{F}_{0}$. We assumed that the joint distribution of the vector $\left(X_{0}, W_{t_{1}}, \ldots, W_{t_{N}}\right)$ is known and therefore can be simulated. These results proved that one could use simulations to study the path and probabilistic properties of such anticipating processes.

Nevertheless, the proof of weak approximation was complicated and required stringent conditions on the random variable $X_{0}$. Here the problem comes from the fact that although $\Phi_{t}\left(X_{0}\right)$ satisfies a stochastic differential equation of anticipative type, there is no partial differential equation associated to it. Furthermore, the Markov property is not satisfied.

In this article we propose to continue this study. We want to concentrate our efforts on ordinary stochastic differential equations with boundary conditions. As many high order sde's with boundary conditions can be reduced to first order ones, we will start studying the weak approximations for first order stochastic differential equations with boundary conditions. Later we will handle the second order equations.

Here, we consider approximations for the density of the solution to a sde with boundary conditions. These equations arise naturally as extensions of ordinary differential equations with boundary conditions. The solutions of such equations can be written as $\Phi_{t}\left(X_{0}\right)$. There are various added complications to the definition and the analysis of approximations in this case.

First, one has to approximate $X_{0}$ through a procedure that resembles the classical shooting method. This method is not well defined in the whole sample space; therefore we will need a localization procedure. Second, the approximation to $X_{0}$ does not satisfy the requirements of the weak approximation results in [2]. Third, we are interested in approximating the density of a process with the possible complication that the approximating process may not have a density in itself, although the limit may have one.

To solve these problems, we propose a variant of the classical proof of weak rate of convergence.

In order to give a clear proof of our final goal (see Theorem4.3), we will gradually introduce this modification to the classical proof. The final goal is to find rates of convergence for approximations to the densities of random variables of the type $\Phi_{t}\left(X_{0}\right)$.

First, we study the case when $X_{0}$ is a random variable such that the joint law of $\left(X_{0}, W_{t_{1}}, \ldots, W_{t_{N}}\right)$ is known beforehand. Although this is not the case for sde's with boundary conditions, this will be an important step towards our final goal. In the second step, we study the case when $X_{0}$ also has to be approximated: first, in the case $X_{0}$ is generated by another diffusion, and then, in the case of stochastic differential equations with boundary conditions.

Instead of using the Markov property and $u(t, x)$, we use the integration by parts of the Malliavin calculus. Therefore the variant we introduce to prove these 
results requires the use of flow properties, techniques of Malliavin calculus and the smoothness of the processes involved.

With these tools we will prove weak approximation results of the type (4) for the cases mentioned above. Furthermore, one can also obtain extensions when $f$ belongs to a wider class that includes the indicator function and the delta function. This type of results will provide a way to approximate the distribution and density functions of the processes involved through the use of appropiate Monte Carlo methods.

The study of approximations for density functions has the added difficulty that one has to show that the Malliavin covariance matrix of the approximating process is uniformly bounded with respect to the step size. We prove in the Appendix that something close to this happens (see Lemma 7.2), which will be enough for our approximation result.

After some preliminaries we will discuss in Section 3 our method of proof for weak approximations in the case of diffusions composed with an anticipating random variable. In this section we suppose that $X_{0}$ is a smooth random variable in the Malliavin sense. Then we apply this result to the case when $X_{0}$ is the final point of another diffusion. This example provides a first case where the initial random variable also needs to be approximated.

Then we start to consider our approach in a more difficult anticipative setting such as in the case of stochastic differential equations with boundary conditions. This study is carried out in Section 4 and is divided into two parts, first for the one dimensional case (Section 4.1) and then in the general multidimensional case (Section 4.2).

In the first, the approximation can be considered as the natural generalization of the shooting method for ordinary differential equations with boundary conditions. Here, we have the added complication that a localization technique is needed. We show that our method of proof also works under the appropiate localization. Therefore, the main difference with Section 3 is the fact that $X_{0}$ is only locally smooth and that it also has to be approximated. Finally we consider the approximation of densities for the one dimensional case. In this case, besides the localization procedure, we also need to start considering when the density of the solution to the sde with boundary conditions exists. This involves further calculations related to the Malliavin covariance matrix, and the added problem of considering a nondegenerate approximation.

At the beginning of Section 4 we also give a brief introduction to stochastic differential equations with boundary conditions.

Then in Section [5 we give a short description of a possible real application of these equations together with a general result of diffusion approximation. We finish with some conclusions and possible generalizations of our method. At the end of this article, in Section 7, we have collected a series of auxiliary results that are used throughout the text.

In this article, $C$ will denote positive constants that may change from one line to the next. These constants are always assumed independent of $\delta$ and the partition $\pi$ unless it is explicitly stated otherwise. 


\section{Preliminaries}

Now we introduce some basic tools from Malliavin calculus that will be used throughout the text. For further reference see [23]. Let $(\Omega, \mathcal{F}, P)$ be the canonical Wiener space which supports a $d$-dimensional Wiener process $W$. Let $C_{b}^{\infty}\left(\mathbb{R}^{d n}\right)$ be the set of $C^{\infty}$ functions $f: \mathbb{R}^{n d} \rightarrow \mathbb{R}$ which are bounded and have bounded derivatives of all orders. The class of real random variables of the form $f\left(W_{t_{1}}, \ldots, W_{t_{n}}\right)$, $f \in C_{b}^{\infty}\left(\mathbb{R}^{n d}\right), t_{1}, \ldots, t_{n} \geq 0$ (the class of infinitely differentiable functions with bounded derivatives), is denoted by $\mathcal{S} . \mathbb{D}^{1, p}$ designates the Banach space which is the completion of $\mathcal{S}$ with respect to the norm

$$
\|F\|_{1, p}=\left\{E|F|^{p}\right\}^{1 / p}+\left(\sum_{j=1}^{d} E\left[\left\{\int_{0}^{1}\left|D_{s}^{j} F\right|^{2} d s\right\}^{p / 2}\right]\right)^{1 / p},
$$

where $\left(\mathbb{I}_{A}\right.$ denotes the indicator function of the set $\left.A\right)$

$$
D_{s}^{j} F=\sum_{i=1}^{n} \frac{\partial f}{\partial x_{j i}}\left(W_{t_{1}}, \ldots, W_{t_{d}}\right): \mathbb{I}_{\left[0, t_{i}\right]}(s) .
$$

$\mathbb{D}^{\alpha, p}$ is defined analogously, and its associated norm is denoted by $\|\cdot\|_{\alpha, p}$. Also, let $\mathbb{D}^{\alpha, \infty}=\bigcap_{p \geq 1} \mathbb{D}^{\alpha, p}$ and $\mathbb{D}^{\infty}=\bigcap_{p \geq 1} \cap_{\alpha \geq 1} \mathbb{D}^{\alpha, p}$. The localization of $\mathbb{D}^{\alpha, p}$ is denoted by $\mathbb{D}_{\text {loc }}^{\alpha, p}$. That is, $\mathbb{D}_{\text {loc }}^{\alpha, p}$ is the set of random variables $F$ such that there exists a sequence $\left\{\left(\Omega^{\epsilon}, F^{\epsilon}\right), 0<\epsilon<1\right\} \subset \mathcal{F} \times \mathbb{D}^{\alpha, p}$ such that

(i) $\Omega^{\epsilon} \uparrow \Omega$, a.s.

(ii) $F=F^{\epsilon}$ a.s. on $\Omega^{\epsilon}$.

Then one defines $D_{s_{1}}^{j_{1}} \cdots D_{s_{k}}^{j_{k}} F=D_{s_{1}}^{j_{1}} \cdots D_{s_{k}}^{j_{k}} F^{\epsilon}$ on $\Omega^{\epsilon}$ for $k \leq \alpha, j_{1}, \ldots, j_{k} \in$ $\{1, \ldots, d\}$, and we say that $\left(\Omega^{\epsilon}, F^{\epsilon}\right)$ localizes $F$.

The adjoint of the closed unbounded operator $D: \mathbb{D}^{1,2} \rightarrow L^{2}([0,1] \times \Omega)$ is usually denoted by $\delta$ and is called the Skorohod integral. Its domain can be characterized as the set of measurable processes $u \in L^{2}([0,1] \times \Omega)$ such that there exists a positive constant $C$ (which may depend on $u$ ) such that

$$
\left|E\left(\int_{0}^{1} D_{t} F u_{t} d t\right)\right| \leq C\|F\|_{2}
$$

for all $F \in \mathbb{D}^{1,2}$. Then the Skorohod integral, for $u$ an element of its domain, is the square integrable random variable determined by the duality relation

$$
E(\delta(u) F)=E\left(\int_{0}^{1} D_{t} F u_{t} d t\right)
$$

for all $F \in \mathbb{D}^{1,2}$. The Skorohod integral turns out to be an extension of the classical Itô integral, and it allows the integration of processes that are not necessarily adapted.

In order to avoid confusion we will use $D$ for the derivative defined above, and $\nabla$ or the ' notation for classical derivatives of functions.

When considering densities of random variables we will use the concept of Malliavin covariance matrix. For this, define for $F \in\left(\mathbb{D}^{1,1}\right)_{l o c}^{m}$ the Malliavin covariance matrix of $F$ as $\Delta_{F}^{i j}=\left\langle D F^{i}, D F^{j}\right\rangle_{L^{2}[0,1]}$. If $F \in \mathbb{D}^{\infty}$ and $\operatorname{det} \Delta_{F}^{-1} \in \bigcap_{p>1} L^{p}(\Omega)$, then $F$ has a smooth density. 
A measurable process $u$ with integrable paths a.s. is said to be Stratonovich integrable with respect to $W^{j}$ if for any increasing sequence of partitions $\left\{\Pi_{n}=\right.$ $\left.\left\{0=t_{0}^{n}<\cdots<t_{n}^{n}=1\right\}\right\}$ of $[0, T]$, such that $\left|\Pi_{n}\right| \rightarrow 0$, the sequence

$$
\sum_{i=0}^{n-1} \frac{1}{t_{i+1}^{n}-t_{i}^{n}}\left(\int_{t_{i}^{n}}^{t_{i+1}^{n}} u_{s} d s\right)\left(W_{t_{i+1}^{n}}^{j}-W_{t_{i}^{n}}^{j}\right)
$$

converges in probability. In such a case we will denote the limit by $\int_{0}^{1} u_{t} \circ d W_{t}^{j}$. It is well-known that if $u \in \mathbb{L}_{C, l o c}^{1,2}$ (for a definition of this space, see [23]), then $u$ is Stratonovich integrable with respect to $W^{j}$, for $j \in\{1, \ldots, k\}$.

Now we give some notation related to stochastic differential equations. From here onwards we reduce our study to one dimensional sde's for convenience.

Let $\Phi_{t}(s, x)$ be the stochastic flow (sometimes also denoted by $\Phi(t, x)$ or $\Phi_{t}(x)$ in the case $s=0$ ), defined as the solution of

$$
\Phi_{t}(s, x)=x+\int_{s}^{t} \sigma\left(\Phi_{u}(s, x)\right) \circ d W_{u}+\int_{s}^{t} b\left(\Phi_{u}(s, x)\right) d u, s \leq t \in[0,1],
$$

where $b, \sigma: \mathbb{R} \rightarrow \mathbb{R}$ are smooth functions with bounded derivatives. Now we define the Euler approximation for $\Phi$. For this, let $\pi=\left\{0=t_{0}<\ldots<t_{N}=1\right\}$ be a partition of $[0,1]$. As before, we define the shift operator $\eta(t)$ and we take $\|\pi\| \leq \delta$. Let $\bar{\Phi}$ denote the Euler-Maruyama scheme, defined by

$$
\bar{\Phi}_{t}(s, x)=x+\int_{s}^{t} \sigma\left(\bar{\Phi}_{\eta(u)}(s, x)\right) d W_{u}+\int_{s}^{t} m\left(\bar{\Phi}_{\eta(u)}(s, x)\right) d u .
$$

Here $m=b+\frac{1}{2} \sigma^{\prime} \sigma$. In the particular case that $s=0$ we simplify the notation and use $\bar{\Phi}_{t}(x)$ and $\Phi_{t}(x)$ instead of $\bar{\Phi}_{t}(0, x)$ and $\Phi_{t}(0, x)$.

We will also use some terminology related to the high order Itô-Taylor formula as stated in [16]. That is, let $\mathcal{M}=\left\{\left(j_{1}, \ldots, j_{l}\right) ; j_{i} \in\{0,1\}, i \in\{1, \ldots, l\}\right.$, for $l=$ $1, \ldots\} \cup\{v\}$ where $v$ denotes the multi-index of length 0 . For a multi-index $\alpha=$ $\left(j_{1}, \ldots, j_{l}\right)$ define the length of $\alpha$ as $l(\alpha)=l$, and define $n(\alpha)$ as the number of zeros in $\alpha,-\alpha=\left(j_{2}, \ldots, j_{l}\right)$ and $\alpha-=\left(j_{1}, \ldots, j_{l-1}\right)$. Then for $f:[0, T] \times \mathbb{R} \rightarrow \mathbb{R}$ we define the following operators:

$$
\begin{gathered}
L^{1} f(s, x)=\sigma(x) \frac{\partial f}{\partial x}(s, x), \\
L^{0} f(s, x)=\frac{\partial f}{\partial s}(s, x)+m(x) \frac{\partial f}{\partial x}(s, x)+\frac{1}{2} \sigma^{2}(x) \frac{\partial^{2} f}{\partial x^{2}}(s, x) .
\end{gathered}
$$

For $\alpha=\left(j_{1}, \ldots, j_{l}\right)$ we define by induction

$$
g_{\alpha}= \begin{cases}g, & l=0 \\ L^{j_{1}} g_{-\alpha}, & l \geq 1\end{cases}
$$

When $g$ is not explicitly stated we shall always take it to be the identity function $g(t, x)=x$. Also, we define the following Wiener functionals for an adapted process $f:$

$$
I_{\alpha}[f(\cdot)]_{s, t}= \begin{cases}f(t), & l=0, \\ \int_{s}^{t} I_{\alpha-}[f(\cdot)]_{s, u} d W_{u}^{j_{l}}, & l \geq 1 .\end{cases}
$$

Here $d W_{u}^{0}=d u$ and $I_{\alpha, s, t}=I_{\alpha}[1]_{s, t}$. Also let $\Gamma_{\beta}=\{\alpha \in \mathcal{M} ; l(\alpha) \leq \beta\}$ and $B\left(\Gamma_{\beta}\right)=\left\{\alpha \in \mathcal{M}-\Gamma_{\beta} ;-\alpha \in \Gamma_{\beta}\right\}$. 
Also, the space of differentiable functions with polynomial growth at infinity is defined for $k=0,1, \ldots$ as

$$
\begin{aligned}
C_{p}^{k}\left(\mathbb{R}^{d}\right) \equiv C_{p}^{k}\left(\mathbb{R}^{d} ; \mathbb{R}\right)= & \left\{f \in C^{k}\left(\mathbb{R}^{d} ; \mathbb{R}\right) ; \quad \exists K \geq 0, r \in \mathbb{N}\right. \\
& \text { such that } \left.\left|\partial_{y}^{j} f(y)\right| \leq K\left(1+|y|^{r}\right), \text { for any } l(j) \leq k, y \in \mathbb{R}\right\} .
\end{aligned}
$$

Here, $j$ denotes a multi-index from $\{1, \ldots, d\}^{l(j)}, l(j)$ denotes its length, and $\partial_{y}^{j} f(y)$ denotes the high order partial derivative of $f$ with respect to the indices in $j$. In the case $d=1$ we write $C_{p}^{k} \equiv C_{p}^{k}(\mathbb{R})$.

\section{WEAK APPROXIMATION FOR THE COMPOSITION OF TWO DIFFUSIONS}

In this section we will study the weak approximation of $\Phi_{t}\left(X_{0}\right)$ by $\bar{\Phi}_{t}\left(X_{0}\right)$, where $X_{0}$ is an appropiate smooth random variable. Therefore we will be assuming that one knows how to simulate $\left(X_{0}, W_{t_{1}}, \ldots, W_{t_{N}}\right)$. Although we work with one dimensional sde's, the results in this section have straightforward generalizations to the multidimensional case.

We start with a preliminary result on the generalized strong rate of convergence of the Euler scheme. This is a necessary step in order to prove the weak rate of convergence.

Proposition 3.1. Assume that, for $k \in \mathbb{N}, X_{0} \in \mathbb{D}^{k, \infty}$ with $\sup _{s \in[0,1]} E\left|D_{s} X_{0}\right|^{p}<$ $\infty$ for all $p>1$. Then $\Phi_{t}\left(X_{0}\right), \bar{\Phi}_{t}\left(X_{0}\right) \in \mathbb{D}^{k, \infty}$, and furthermore there exists a positive constant $C(k, p)$, depending on $p$ and $k$, such that

$$
\left\|\Phi_{t}\left(X_{0}\right)-\bar{\Phi}_{t}\left(X_{0}\right)\right\|_{k, p} \leq C(k, p) \delta^{\frac{1}{2}} .
$$

Proof. $\Phi_{t}\left(X_{0}\right), \bar{\Phi}_{t}\left(X_{0}\right) \in \mathbb{D}^{k, \infty}$ are proved using Lemmas 7.37 .4 in the Appendix and the chain rule for the Malliavin calculus.

We assume in the rest of the proof that $k=1$; the general case is left for the reader. In this case, one needs to estimate

$$
\begin{aligned}
\left\|\Phi_{t}\left(X_{0}\right)-\bar{\Phi}_{t}\left(X_{0}\right)\right\|_{1, p}= & \left\{E\left|\Phi_{t}\left(X_{0}\right)-\bar{\Phi}_{t}\left(X_{0}\right)\right|^{p}\right\}^{1 / p} \\
& +\left(E\left[\left\{\int_{0}^{1}\left|D_{s}\left(\Phi_{t}\left(X_{0}\right)-\bar{\Phi}_{t}\left(X_{0}\right)\right)\right|^{2} d s\right\}^{p / 2}\right]\right)^{1 / p} .
\end{aligned}
$$

First, due to Lemma 7.3 in the Appendix, there exists a positive constant $C(p)$ such that

$$
E\left|\Phi_{t}\left(X_{0}\right)-\bar{\Phi}_{t}\left(X_{0}\right)\right|^{p} \leq C(p) \delta^{\frac{p}{2}}
$$

It is not difficult to compute $D_{s} \Phi_{t}\left(X_{0}\right)$ and $D_{s} \bar{\Phi}_{t}\left(X_{0}\right)$. This gives, for $s \leq t$,

$$
\begin{aligned}
D_{s}\left(\Phi_{t}\left(X_{0}\right)\right) & =D_{s}\left(\Phi_{t}\right)\left(X_{0}\right)+\nabla\left(\Phi_{t}\right)\left(X_{0}\right) D_{s} X_{0} \\
& =\nabla\left(\Phi_{t}\right)\left(s, \Phi_{s}\left(X_{0}\right)\right) \sigma\left(\Phi_{s}\left(X_{0}\right)\right)+\nabla\left(\Phi_{t}\right)\left(X_{0}\right) D_{s} X_{0} .
\end{aligned}
$$

Analogously, for $s \leq t$

$$
D_{s}\left(\bar{\Phi}_{t}\left(X_{0}\right)\right)=\nabla\left(\bar{\Phi}_{t}\right)\left(\eta_{1}(s), \bar{\Phi}_{\eta_{1}(s)}\left(X_{0}\right)\right) \sigma\left(\bar{\Phi}_{\eta(s)}\left(X_{0}\right)\right)+\nabla\left(\Phi_{t}\right)\left(X_{0}\right) D_{s} X_{0} .
$$


Here, $\eta_{1}(s):=\min \left\{t_{k} ; t_{k} \geq s\right\}$ and $\nabla\left(\bar{\Phi}_{t}\right)\left(\eta_{1}(s), x\right)=1$ if $s \leq t<\eta_{1}(s)$. Now consider

$$
\begin{aligned}
E\left(\mid D_{s}(\right. & \left.\left.\Phi_{t}\left(X_{0}\right)-\bar{\Phi}_{t}\left(X_{0}\right)\right)\left.\right|^{p}\right) \\
\leq & C(p)\left\{E\left(\left|\nabla\left(\Phi_{t}\right)\left(s, \Phi_{s}\left(X_{0}\right)\right) \sigma\left(\Phi_{s}\left(X_{0}\right)\right)-\nabla\left(\bar{\Phi}_{t}\right)\left(s, \bar{\Phi}_{s}\left(X_{0}\right)\right) \sigma\left(\bar{\Phi}_{s}\left(X_{0}\right)\right)\right|^{p}\right)\right. \\
& +E\left(\left|\nabla\left(\bar{\Phi}_{t}\right)\left(s, \bar{\Phi}_{s}\left(X_{0}\right)\right) \sigma\left(\bar{\Phi}_{s}\left(X_{0}\right)\right)-\nabla\left(\bar{\Phi}_{t}\right)\left(s, \bar{\Phi}_{s}\left(X_{0}\right)\right) \sigma\left(\bar{\Phi}_{\eta(s)}\left(X_{0}\right)\right)\right|^{p}\right) \\
& +E\left(\left|\left\{\nabla\left(\bar{\Phi}_{t}\right)\left(s, \bar{\Phi}_{s}\left(X_{0}\right)\right)-\nabla\left(\bar{\Phi}_{t}\right)\left(\eta_{1}(s), \bar{\Phi}_{s}\left(X_{0}\right)\right)\right\} \sigma\left(\bar{\Phi}_{\eta(s)}\left(X_{0}\right)\right)\right|^{p}\right) \\
& +E\left(\left|\left\{\nabla\left(\bar{\Phi}_{t}\right)\left(\eta_{1}(s), \bar{\Phi}_{s}\left(X_{0}\right)\right)-\nabla\left(\bar{\Phi}_{t}\right)\left(\eta_{1}(s), \bar{\Phi}_{\eta_{1}(s)}\left(X_{0}\right)\right)\right\} \sigma\left(\bar{\Phi}_{\eta(s)}\left(X_{0}\right)\right)\right|^{p}\right) \\
& \left.+E\left(\left|\nabla\left(\Phi_{t}\right)\left(X_{0}\right)-\nabla\left(\bar{\Phi}_{t}\right)\left(X_{0}\right)\right|^{p}\left|D_{s} X_{0}\right|^{p}\right)\right\} \\
= & e_{1}+e_{2}+e_{3}+e_{4}+e_{5} .
\end{aligned}
$$

We have $\sup _{s \in[0, t]}\left(e_{1}+e_{2}+e_{4}+e_{5}\right) \leq C(p) \delta^{p / 2}$, due to Lemma7.4 in the Appendix.

To find the rate of convergence to 0 of $e_{3}$ it is enough to note that the processes $\alpha_{t}=\bar{\Phi}_{t}(s, x)-\bar{\Phi}_{t}\left(\eta_{1}(s), x\right)$ and $\beta_{t}=\nabla \bar{\Phi}_{t}(s, x)-\nabla \bar{\Phi}_{t}\left(\eta_{1}(s), x\right)$ are the solution of linear stochastic differential equations. For example, $\alpha_{t}$ solves the following equation:

$$
\begin{aligned}
\alpha_{t}= & \int_{s}^{\eta_{1}(s)} m\left(\bar{\Phi}_{\eta(u)}(s, x)\right) d u+\int_{s}^{\eta_{1}(s)} \sigma\left(\bar{\Phi}_{\eta(u)}(s, x)\right) d W_{u} \\
& +\int_{\eta_{1}(s)}^{t} \int_{0}^{1} \nabla m\left((1-v) \bar{\Phi}_{\eta(u)}\left(\eta_{1}(s), x\right)+v \bar{\Phi}_{\eta(u)}(s, x)\right) d v \alpha_{u} d u \\
& +\int_{\eta_{1}(s)}^{t} \int_{0}^{1} \nabla \sigma\left((1-v) \bar{\Phi}_{\eta(u)}\left(\eta_{1}(s), x\right)+v \bar{\Phi}_{\eta(u)}(s, x)\right) d v \alpha_{u} d W_{u} .
\end{aligned}
$$

From here it follows using Gronwall's Lemma that $E\left(\sup _{0 \leq t \leq 1}\left|\alpha_{t}\right|^{p}\right) \leq C(p) \delta^{p / 2}$. In the same manner one proves that $\beta_{t}$ satisfies the same property. Then one can conclude that $e_{3} \leq C(p) \delta^{p / 2}$.

This proof can actually be used to prove even stronger statements than those proven here (see Lemma 7.4 in the Appendix).

Theorem 3.1. Let $\pi$ be a partition of size $\delta$, and let $\bar{\Phi}$ be defined as in (6). Suppose that $f \in C_{p}^{4}(\mathbb{R})$. Also assume that $b$ and $\sigma$ are smooth functions with bounded derivatives. Finally, suppose that $X_{0} \in \mathbb{D}^{2, \infty}$ and

$$
\sup _{s} E\left|D_{s} X_{0}\right|^{4}<\infty, \quad \sup _{s, u} E\left|D_{s} D_{u} X_{0}\right|^{2}<\infty
$$

Then for any $t \in[0,1]$, there exists a positive constant $C$ that depends on $f$ such that

$$
\left|E\left[f\left(\Phi_{t}\left(X_{0}\right)\right)-f\left(\bar{\Phi}_{t}\left(X_{0}\right)\right)\right]\right| \leq C \delta .
$$


Proof. First assume without loss of generality that $t \in \pi$. We then have

(8)

$$
\begin{aligned}
& E f\left(\bar{\Phi}_{t}\left(X_{0}\right)\right)-E f\left(\Phi_{t}\left(X_{0}\right)\right) \\
&=\sum_{i=1}^{n(t)} E\left[f\left(\Phi_{t}\left(t_{i}, \bar{\Phi}_{t_{i}}\left(X_{0}\right)\right)\right)-f\left(\Phi_{t}\left(t_{i-1}, \bar{\Phi}_{t_{i-1}}\left(X_{0}\right)\right)\right)\right] \\
&=\sum_{i=1}^{n(t)} E\left[f\left(\Phi_{t}\left(t_{i}, \bar{\Phi}_{t_{i}}\left(X_{0}\right)\right)\right)-f\left(\Phi_{t}\left(t_{i}, \bar{\Phi}_{t_{i-1}}\left(X_{0}\right)\right)\right)\right. \\
&\left.\quad+f\left(\Phi_{t}\left(t_{i}, \bar{\Phi}_{t_{i-1}}\left(X_{0}\right)\right)\right)-f\left(\Phi_{t}\left(t_{i}, \Phi_{t_{i}}\left(t_{i-1}, \bar{\Phi}_{t_{i-1}}\left(X_{0}\right)\right)\right)\right]\right] \\
&=\sum_{i=1}^{n(t)} E\left[\left.\frac{\partial}{\partial x} f\left(\Phi_{t}\left(t_{i}, x\right)\right)\right|_{x=\bar{\Phi}_{t_{i-1}}\left(X_{0}\right)}\right. \\
& \quad \times\left\{\bar{\Phi}_{t_{i}}\left(X_{0}\right)-\bar{\Phi}_{t_{i-1}}\left(X_{0}\right)-\left(\Phi_{t_{i}}\left(t_{i-1}, \bar{\Phi}_{t_{i-1}}\left(X_{0}\right)\right)-\bar{\Phi}_{t_{i-1}}\left(X_{0}\right)\right)\right\} \\
&\left.+R_{i}\left(\bar{\Phi}_{t_{i}}\left(X_{0}\right)\right)-R_{i}\left(\Phi_{t_{i}}\left(t_{i-1}, \bar{\Phi}_{t_{i-1}}\left(X_{0}\right)\right)\right)\right]
\end{aligned}
$$

where $n(t)=\max \left\{j ; t_{j} \leq t\right\}$ and

$$
R_{i}(U)=\int_{0}^{1} \int_{0}^{s_{2}} \frac{\partial^{2}}{\partial x^{2}} f\left(\Phi_{t}\left(t_{i}, \cdot\right)\right)\left(U+s_{1}\left(U-\bar{\Phi}_{t_{i-1}}\left(X_{0}\right)\right)\right)\left(U-\bar{\Phi}_{t_{i-1}}\left(X_{0}\right)\right)^{2} d s_{1} d s_{2} .
$$

To shorten the proof we will select some terms to show how the proof is done.

For example, in (8) one has to analyze the term (this is a particular form of the Itô-Taylor formula, see Theorem 5.5.1 in [16])

$$
\begin{array}{r}
\left(\bar{\Phi}_{t_{i}}\left(X_{0}\right)-\bar{\Phi}_{t_{i-1}}\left(X_{0}\right)-\left(\Phi_{t_{i}}\left(t_{i-1}, \bar{\Phi}_{t_{i-1}}\left(X_{0}\right)\right)-\bar{\Phi}_{t_{i-1}}\left(X_{0}\right)\right)\right) \\
=\left.\sum_{\alpha \in B\left(\Gamma_{1}\right)} I_{\alpha, t_{i-1}, t_{i}}\left(g_{\alpha}\left(\cdot, \Phi .\left(t_{i-1}, \bar{\Phi}_{t_{i-1}}(x)\right)\right)\right)\right|_{x=X_{0}} .
\end{array}
$$

Here $g(x)=x$ and $g_{(0,1)}$ is defined in (7). Some of these terms will involve stochastic integrals that have to be plugged into (8). For example, the expectation of one of these terms will generate, for $\alpha=(0,1)$,

$$
E\left\{\left.\left.\frac{\partial}{\partial x} f\left(\Phi_{t}\left(t_{i}, x\right)\right)\right|_{x=\bar{\Phi}_{t_{i-1}}\left(X_{0}\right)} I_{(0,1), t_{i-1}, t_{i}}\left(g_{(0,1)}\left(\cdot, \Phi_{.}\left(t_{i-1}, \bar{\Phi}_{t_{i-1}}(x)\right)\right)\right)\right|_{x=X_{0}}\right\} .
$$

Next, this equals

$$
\int_{t_{i-1}}^{t_{i}} E\left(\left.\left.\frac{\partial}{\partial x} f\left(\Phi_{t}\left(t_{i}, x\right)\right)\right|_{x=\Phi_{t_{i-1}}\left(X_{0}\right)} \int_{t_{i-1}}^{s} G\left(\Phi_{u}\left(t_{i-1}, \bar{\Phi}_{t_{i-1}}(x)\right)\right) d W_{u}\right|_{x=X_{0}}\right) d s,
$$


where $G(x)=m(x) \sigma^{\prime}(x)+\frac{1}{2} \sigma^{2}(x) \sigma^{\prime \prime}(x)$. Then, applying (51) we see that the above expression becomes

$$
\begin{aligned}
\int_{t_{i-1}}^{t_{i}} \int_{t_{i-1}}^{s} E\{ & \left.\frac{\partial}{\partial x} f\left(\Phi_{t}\left(t_{i}, x\right)\right)\right|_{x=\bar{\Phi}_{t_{i-1}}\left(X_{0}\right)} D_{u} X_{0} \frac{\partial G\left(\Phi_{u}\left(t_{i-1}, \bar{\Phi}_{t_{i-1}}(\cdot)\right)\right)}{\partial x}\left(X_{0}\right) \\
& \left.+\left.D_{u} \frac{\partial}{\partial x} f\left(\Phi_{t}\left(t_{i}, x\right)\right)\right|_{x=\Phi_{t_{i-1}}\left(X_{0}\right)} G\left(\Phi_{u}\left(t_{i-1}, \bar{\Phi}_{t_{i-1}}\left(X_{0}\right)\right)\right)\right\} d u d s
\end{aligned}
$$

To finish one obtains (using Lemmas 7.3 and 7.4 ) that the above integrand is smaller than $C \delta^{2}$ uniformly in $\left\{(s, u) \in\left[t_{i-1}, t_{i}\right]^{2} ; \quad u \leq s\right\}$. Here $C$ is a positive constant independent of the partition $\pi, \delta, s$ and $t$. The treatment of the other terms in (10) is similar.

For example, we will bound in the first term in (12). That is, we will prove that there exists a positive constant, independent of the partition $\pi$ and $\delta$, such that

$$
\sup _{u \in\left[t_{i-1}, t_{i}\right]}\left|E\left(\left.D_{u} X_{0} \frac{\partial}{\partial x} f\left(\Phi_{t}\left(t_{i}, x\right)\right)\right|_{x=\bar{\Phi}_{t_{i-1}}\left(X_{0}\right)} \frac{\partial G\left(\Phi_{u}\left(t_{i-1}, \bar{\Phi}_{t_{i-1}}(\cdot)\right)\right)}{\partial x}\left(X_{0}\right)\right)\right|<C .
$$

In fact, we have $D_{u} X_{0} \in L^{p}(\Omega)$, for any $p \leq 2$, uniformly in $u$ by hypothesis. As $f$ has polynomial growth at infinity, and using flow properties together with Lemma 7.3, we also have

$$
\left.\frac{d}{d x} f\left(\Phi_{t}\left(t_{i}, x\right)\right)\right|_{x=\Phi_{t_{i-1}}\left(X_{0}\right)} \in L^{p}(\Omega)
$$

for any $p>1$ uniformly in $\left\{\left(t_{i}, t_{i-1}\right) \in[0, t]^{2} ; \quad t_{i-1}<t_{i}\right\}$.

Now note that $G(x)$ is smooth with polynomial growth at infinity. Therefore we have as before that $\frac{\partial}{\partial x} G\left(\Phi_{u}\left(t_{i-1}, \bar{\Phi}_{t_{i-1}}\left(X_{0}\right)\right)\right) \in L^{p}(\Omega)$, for any $p>1$ uniformly in $t_{i-1} \in[0, t]$. Therefore the result follows.

Remark 3.1. 1. When dealing with the residues in (9) it is necessary to repeat some of the steps shown in the proof above. In fact, for example we will have a term of the type

$$
\left(\bar{\Phi}_{t_{i}}-\bar{\Phi}_{t_{i-1}}\left(X_{0}\right)\right)^{2}=\left(m\left(\bar{\Phi}_{t_{i-1}}\left(X_{0}\right)\right)\left(t_{i}-t_{i-1}\right)+\sigma\left(\bar{\Phi}_{t_{i-1}}\left(X_{0}\right)\right)\left(W_{t_{i}}-W_{t_{i-1}}\right)\right)^{2} .
$$

Here one expands the square and again uses (51) whenever increments of the Wiener process appear.

2. The condition $\sup _{s, u} E\left|D_{s} D_{u} X_{0}\right|^{2}<\infty$ is used in (13) in the case $\alpha=(1,1)$. 
3. Note that the conditions on the derivatives of $X_{0}$ can be relaxed if one uses the appropiate Hölder inequalities in (13). This weaker condition is of the form: There exists $\epsilon>0$ such that

$$
\sup _{s \in[0,1]} E\left|D_{s} X_{0}\right|^{2+\epsilon}+\sup _{s, u \in[0,1]} E\left|D_{s} D_{u} X_{0}\right|^{1+\epsilon}<\infty .
$$

4. Theorem 3.1 is an improvement of Theorem 4.2 in [2]. The method of proof is different. Theorem 4.2 in [2] is strongly based in some generator of a highly complex process which in spirit resembles the classical proofs that one can find in e.g. [16], Chapter 14.

We will now give a first application of this theorem. Consider the weak approximation problem for $f\left(\Phi_{t}\left(X_{0}\right)\right)$, where $X_{0}$ is generated by a diffusion. Up to the previous theorem it was assumed that the vector $\left(X_{0}, W_{t_{1}}, \ldots, W_{t_{N}}\right)$ had a known joint law that can be simulated. Now we consider the case when $X_{0}$ also needs to be approximated through an Euler-Maruyama approximation. For this, let $Z$ be the diffusion defined by

$$
Z_{t}=Z_{0}+\int_{0}^{t} B\left(Z_{s}\right) d W_{s}+\int_{0}^{t} A\left(Z_{s}\right) d s
$$

Here $Z_{0}$ is any $\mathcal{F}_{0}$-measurable random variable in $L^{p}(\Omega)$ for any $p>1$. Analogously define the Euler approximation scheme $\bar{Z}$ for $Z$ using a partition $\pi^{\prime}:=\left\{0=s_{0}<\right.$ $\left.\cdots<s_{M}=1\right\}$ with $\left|\pi^{\prime}\right| \leq \delta^{\prime}$. Note that the noise that generates $Z$ is the same to the noise that generates $X$. Therefore, in general, $X$ and $Z$ are not independent.

Assume that $A$ and $B$ are smooth with bounded derivatives. Then $Z_{1} \in \mathbb{D}^{2, \infty}$ and, furthermore,

$$
\sup _{s_{1}, \ldots, s_{k}} E\left[\sup _{t}\left|D_{s_{1}} \ldots D_{s_{k}}\left(Z_{t}-\bar{Z}_{t}\right)\right|^{p}\right] \leq C_{k}\left(\delta^{\prime}\right)^{\frac{p}{2}}
$$

for any $k \in \mathbb{N}$ (this is done using essentially the same proof as in Proposition 3.1). Therefore by Theorem 3.1 we obtain that if $f \in C_{p}^{3}$ there exists a positive constant $C$, that may depend on $f$ and $t$ but it is independent of $\pi, \pi^{\prime}, \delta$ and $\delta^{\prime}$, such that

$$
\left|E f\left(\Phi_{t}\left(Z_{1}\right)\right)-E f\left(\bar{\Phi}_{t}\left(Z_{1}\right)\right)\right| \leq C \delta .
$$

A realistic case is to consider that $Z_{1}$ cannot be simulated exactly, and that we have to use an Euler approximation of it. The following lemma will give a weak approximation result for $\Phi_{t}\left(Z_{1}\right)$.

Lemma 3.1. Let $f \in C_{p}^{4}(\mathbb{R})$. Assume that $A, B, b$ and $\sigma$ are smooth with bounded derivatives. Then there exists a positive constant $C$, that may depend on $f$ and $t$, such that

$$
\left|E f\left(\bar{\Phi}_{t}\left(Z_{1}\right)\right)-E f\left(\bar{\Phi}_{t}\left(\bar{Z}_{1}\right)\right)\right| \leq C \delta^{\prime} .
$$


Proof. We start as in the proof of Theorem 3.1 Denote by $\phi_{t}(x)$ the stochastic flow defined by (14), and assume without loss of generality that $s_{M}=1$. We have

$$
\begin{gathered}
E f\left(\bar{\Phi}_{t}\left(\bar{Z}_{1}\right)\right)-E f\left(\bar{\Phi}_{t}\left(Z_{1}\right)\right) \\
=\sum_{i=1}^{M} E\left[f\left(\bar{\Phi}_{t}\left(\phi_{1}\left(s_{i}, \bar{Z}_{s_{i}}\right)\right)\right)-f\left(\bar{\Phi}_{t}\left(\phi_{1}\left(s_{i-1}, \bar{Z}_{s_{i-1}}\right)\right)\right)\right] \\
=\sum_{i=1}^{M} E\left[f\left(\bar{\Phi}_{t}\left(\phi_{1}\left(s_{i}, \bar{Z}_{s_{i}}\right)\right)\right)-f\left(\bar{\Phi}_{t}\left(\phi_{1}\left(s_{i}, \bar{Z}_{s_{i-1}}\right)\right)\right)\right. \\
\left.\quad+f\left(\bar{\Phi}_{t}\left(\phi_{1}\left(s_{i}, \bar{Z}_{s_{i-1}}\right)\right)\right)-f\left(\bar{\Phi}_{t}\left(\phi_{1}\left(s_{i}, \phi_{s_{i}}\left(s_{i-1}, \bar{Z}_{s_{i-1}}\right)\right)\right)\right)\right] \\
=\sum_{i=1}^{M} E\left[\left.\frac{\partial}{\partial x} f\left(\bar{\Phi}_{t}\left(\phi_{1}\left(s_{i}, x\right)\right)\right)\right|_{x=\bar{Z}_{s_{i-1}}}\right. \\
\times\left(\bar{Z}_{s_{i}}-\bar{Z}_{s_{i-1}}-\left(\phi_{s_{i}}\left(s_{i-1}, \bar{Z}_{s_{i-1}}\right)-\bar{Z}_{s_{i-1}}\right)\right) \\
\left.+R_{i}\left(\bar{Z}_{s_{i}}\right)-R_{i}\left(\phi_{s_{i}}\left(s_{i-1}, \bar{Z}_{s_{i-1}}\right)\right)\right]
\end{gathered}
$$

where

$$
R_{i}(U)=\int_{0}^{1} \int_{0}^{\theta_{2}} \frac{\partial^{2}}{\partial x^{2}} f\left(\bar{\Phi}_{t}\left(\phi_{1}\left(s_{i}, x\right)\right)\right)\left(U+\theta_{1}\left(U-\bar{Z}_{s_{i-1}}\right)\right)\left(U-\bar{Z}_{s_{i-1}}\right)^{2} d \theta_{1} d \theta_{2} .
$$

From here we can continue with the argument as in the proof of Theorem 3.1 (in particular, the argument after (8)). The result follows.

Therefore we see that if one approximates the initial condition with a degree of accuracy $\delta$, the new approximation $\bar{\Phi}_{t}\left(\bar{Z}_{1}\right)$ is also of order at least $\delta$.

Note that in the proof of this theorem it was essential that $Z$ is a diffusion in order to be able to use the Itô-Taylor formula as in Chapter 14 of [16]. This will not be the case in the next section.

Summarizing the previous calculations, we have the following result.

Theorem 3.2. Assume that $f \in C_{p}^{4}\left(\mathbb{R}^{d}\right), A, B, b$ and $\sigma$ are smooth with bounded derivatives. Then there exists a positive constant $C$ that depends on $t$ and $f$ but is independent of the partitions $\pi$ and $\pi^{\prime}$ as well as $\delta$ and $\delta^{\prime}$, and

$$
\left|E\left[f\left(\Phi_{t}\left(Z_{1}\right)\right)-f\left(\bar{\Phi}_{t}\left(\bar{Z}_{1}\right)\right)\right]\right| \leq C\left(\delta+\delta^{\prime}\right) .
$$

One can also extend this result to high order weak approximations. A previous result of this type was quoted in [2], Theorem 4.2. In that result the hypotheses on $X_{0}$ were much more restrictive than the ones presented here. In particular, it was required that some type of trace for $D_{t} D_{t} X_{0}$ existed. The argument used approximations for $X_{0}$ that belonged to $\mathcal{S}$.

\section{An Euler type SCHEME FOR SOlutions}

OF STOCHASTIC DIFFERENTIAL EQUATIONS WITH BOUNDARY CONDITIONS

Stochastic differential equations with boundary conditions arise naturally in the study of perturbations of ordinary differential equations with boundary conditions 
as well as stochastic models for many physical phenomena. The behaviour and numerical approximation in the ode case is well understood (see e.g. [7] or [15]).

Stochastic differential equations with boundary conditions have a history that may have started with Kwakerwnaak [21]. Later they have been studied by Ocone and Pardoux [27, Nualart and Pardoux [25, Donati and Martin [8], Garnier [13, among others. These are equations of the type

$$
d X_{t}=\sigma\left(X_{t}\right) \circ d W_{t}+b\left(X_{t}\right) d t, \quad 0 \leq t \leq 1,
$$

with a boundary condition of the form

$$
h\left(X_{0}, X_{1}\right)=\bar{h} .
$$

Most of the studies on these equations concern existence and uniqueness of solutions and the Markov field property.

These equations are anticipative in nature due to the boundary condition. Their solutions are evaluations of flows at random variables. That is, the solution to (15)) and (16), when it exists, can be written as $X_{t}=\Phi_{t}\left(X_{0}\right)$, where $\left\{\Phi_{t}(x) t \in[0,1]\right\}$ is the stochastic flow associated with (15) and $X_{0}$ is the unique solution to (16).

A general type of sde with a given anticipating initial condition was first studied by Ocone and Pardoux [28. They proved existence and uniqueness for solutions by means of an Itô-Ventzell type formula.

Here we are interested in the rate of convergence for a weak approximation to the solution of (15)-(16). That is, we will define an approximation for the solution of (15)-(16) and prove that the approximation converges at some rate to the solution.

We will consider two cases. The first is when the boundary condition is linear and the equation is one dimensional. Existence and uniqueness for this type of equations was considered by Donati and Martin [8].

As in the previous section, we want to find an approximation for $E\left(f\left(\Phi_{t}\left(X_{0}\right)\right)\right)$. Theorem 3.1 is applicable in this case, because $X_{0} \in \mathbb{D}^{\infty}$, as will be stated later. The added complication here is the fact that $X_{0}$ also needs to be approximated. This approximation is not as smooth as in the case studied in Lemma 3.1. In fact, it is only locally smooth, as will be proved in Lemma 4.1. This introduces a new ingredient to this problem, which will be solved through an appropiate localization procedure. We will carry this argument out to analyze approximations of densities for $\Phi_{t}\left(X_{0}\right)$ when they exist.

In the second case we consider a multidimensional equation with $\sigma \equiv B$, a constant matrix and a boundary condition of a general type. In this case one can consider as examples some periodic boundary conditions. Existence and uniqueness was obtained by Nualart and Pardoux [25].

In all the cases considered here it is known (see [25]) that solutions of (15)-(16) are seldom Markov processes. Therefore the idea of using the classical method of analysis (see e.g. Chapter 14 in [16]) through PDE problems cannot be applied here.

In other articles (see [19], [10]), we have considered the necessary preliminaries to study this problem - that is, the existence and smoothness of the density and the strong approximation for the solution of (15)-(16) in the two cases mentioned above. These results are used throughout the text, and we will recall them when necessary.

Although here we only consider approximations for the densities, we could also have considered approximations for the distribution functions. With these results 
in hand one could approximate these type of processes using the appropriate Monte Carlo methods.

4.1. The one dimensional case. In this section we will study approximations for densities of solutions to (15) and (16) in the one dimensional case with linear boundary conditions. Existence and uniqueness of solutions for this case were studied in [8]. That is, let $\sigma$ and $b$ be real functions, and let $F_{0}, F_{1}, h_{0} \in \mathbb{R}$. Consider the equation

$$
\begin{aligned}
& d X_{t}=\sigma\left(X_{t}\right) \circ d W_{t}+b\left(X_{t}\right) d t, \quad 0 \leq t \leq 1, \\
& F_{0} X_{0}+F_{1} X_{1}=h_{0} .
\end{aligned}
$$

Assume from now on that $\sigma$ and $b$ are smooth functions with bounded derivatives and $F_{0} \quad F_{1}>0$. Without loss of generality we will always take $F_{0}>0$ and $F_{1}>0$. In [8] it is proved that under these assumptions, there exists a unique solution to (17) belonging to the space $\mathbb{L}_{C, l o c}^{1, \infty}$. Furthermore, this solution can be written as $\Phi_{t}\left(X_{0}\right)$, where $X_{0}$ is the unique solution to (18).

Next we consider a theorem that states when a smooth density of (17)-(18) exists.

Theorem 4.1 (Kohatsu-Higa and Sanz-Solé [19]). Define

$$
\begin{aligned}
& \Sigma_{0}=\{\sigma\}, \\
& \Sigma_{j}=\left\{[\sigma, V],[b, V]+\frac{1}{2}[\sigma,[\sigma, V]] ; V \in \Sigma_{j-1}\right\}, j \geq 1,
\end{aligned}
$$

where $[\cdot, \cdot]$ denotes the Lie bracket. Suppose that there exist $j_{0} \geq 0$ and $V \in \Sigma_{j_{0}}$ with $\left(V\left(X_{0}\right)\right)^{-1} \in \bigcap_{p \geq 1} L^{p}(\Omega)$. Then, for any $t \in[0,1]$ the law of $X_{t}$ has a $C^{\infty}$ density. Furthermore, there exists a finite positive constant $C_{p}(t)$ such that $\left\|\left(\Delta_{\Phi_{t}\left(X_{0}\right)}\right)^{-1}\right\|_{p} \leq C_{p}(t)$, where $\Delta_{F}$ denotes the Malliavin covariance matrix associated with the random variable $F$.

We will denote the density of $X_{t}$ at the point $y$ by $p(t, y)$.

Let $C:=\left\|\sigma^{\prime}\right\|_{\infty}+\left\|b^{\prime}\right\|_{\infty}$. Choose $\delta, M>0$ satisfying $\delta \vee M<1 / 4 C$, and let $W\left(\Delta_{k}\right)$ denote $W\left(t_{k+1}\right)-W\left(t_{k}\right)$. With this notation one obtains by a recursive argument that $\bar{\Phi}_{t}(x)$ is differentiable in $x$ and that $\bar{\Phi}_{0}^{\prime}(x)=1$,

$$
\bar{\Phi}_{t_{k+1}}^{\prime}(x)=\prod_{i=0}^{k}\left(1+\sigma^{\prime}\left(\bar{\Phi}_{t_{i}}(x)\right) W\left(\Delta_{i}\right)+b^{\prime}\left(\bar{\Phi}_{t_{i}}(x)\right)\left(t_{i+1}-t_{i}\right)\right) .
$$

Then, on the set $L_{M}=\left\{\sup _{0 \leq k \leq N-1}\left|W\left(\Delta_{k}\right)\right|<M\right\}$, one has

$$
\left|\sigma^{\prime}\left(\bar{\Phi}_{t_{i}}(x)\right) W\left(\Delta_{i}\right)+b^{\prime}\left(\bar{\Phi}_{t_{i}}(x)\right)\left(t_{i+1}-t_{i}\right)\right| \leq 1 / 2,
$$

and therefore $\bar{\Phi}_{1}^{\prime}(x)$ is strictly positive. Consequently, if $\omega \in L_{M}$, the function $\bar{G}(x, \omega)$ given by

$$
\bar{G}(x, \omega)=F_{0} x+F_{1} \bar{\Phi}_{1}(x)(\omega)
$$

is monotone. We denote by $\widetilde{X}_{0}(\omega)$ the unique solution to $\bar{G}(x, \omega)=h_{0}, \omega \in L_{M}$. Obviously the probabilty of the set $\bar{L}_{M}$ is small (the bar denotes the complement of the set $\left.L_{M}\right)$. That is, for $q \geq 2$,

$$
P\left(\bar{L}_{M}\right) \leq \frac{C_{q}^{N} \delta^{\frac{q}{2}}}{M^{q}},
$$




$$
\begin{aligned}
C_{q}^{N} & =E\left(\sup _{0 \leq k \leq N-1} \frac{\left|W\left(\Delta_{k}\right)\right|}{\sqrt{\delta}}\right)^{\frac{q}{2}} \\
& =\frac{2}{\pi} \int_{0}^{\infty} v^{q} N\left(\int_{-v}^{v} \exp \left(-\frac{u^{2}}{2}\right) d u\right)^{N-1} \exp \left(-\frac{v^{2}}{2}\right) d v .
\end{aligned}
$$

Furthermore, $\sup _{\delta \in(0,1]} \delta C_{q}^{N}<\infty$.

Remark 4.1. The following procedure provides approximations for $\widetilde{X}_{0}(\omega)$. Assume $F_{0} F_{1}>0$. To simplify the notation we skip the dependence on $\omega$. Fix $\widetilde{X}_{0}^{2,0} \leq \widetilde{X}_{0}^{1,0}$ be such that $\bar{G}\left(\widetilde{X}_{0}^{2,0}\right) \leq h_{0}, \bar{G}\left(\widetilde{X}_{0}^{1,0}\right) \geq h_{0}$. We proceed inductively as follows. Let $\widetilde{X}_{0}^{2, i} \leq \widetilde{X}_{0}^{1, i}$ be such that $\bar{G}\left(\widetilde{X}_{0}^{2, i}\right) \leq h_{0}, \bar{G}\left(\widetilde{X}_{0}^{1, i}\right) \geq h_{0}, i \geq 0$. Consider $\frac{\widetilde{X}_{0}^{2, i}+\widetilde{X}_{0}^{1, i}}{2}$. Then,

$$
\begin{aligned}
& \text { if } \bar{G}\left(\frac{\widetilde{X}_{0}^{2, i}+\widetilde{X}_{0}^{1, i}}{2}\right)<h_{0} \text {, set } \widetilde{X}_{0}^{2, i+1}=\frac{\widetilde{X}_{0}^{2, i}+\widetilde{X}_{0}^{1, i}}{2}, \widetilde{X}_{0}^{1, i+1}=\widetilde{X}_{0}^{1, i}, \\
& \text { if } \bar{G}\left(\frac{\widetilde{X}_{0}^{2, i}+\widetilde{X}_{0}^{1, i}}{2}\right)>h_{0} \text {, set } \widetilde{X}_{0}^{2, i+1}=\widetilde{X}_{0}^{2, i}, \widetilde{X}_{0}^{1, i+1}=\frac{\widetilde{X}_{0}^{2, i}+\widetilde{X}_{0}^{1, i}}{2}, \\
& \text { if } \bar{G}\left(\frac{\widetilde{X}_{0}^{2, i}+\widetilde{X}_{0}^{1, i}}{2}\right)=h_{0} \text {, set } \widetilde{X}_{0}=\frac{\widetilde{X}_{0}^{2, i}+\widetilde{X}_{0}^{1, i}}{2} .
\end{aligned}
$$

Notice that

$$
\left|\widetilde{X}_{0}^{2, i}-\widetilde{X}_{0}^{1, i}\right| \leq \frac{\left|\widetilde{X}_{0}^{2,0}-\widetilde{X}_{0}^{1,0}\right|}{2^{i-1}}, \quad i \geq 1 .
$$

Let $\bar{X}_{0}=\widetilde{X}_{0} \mathbf{1}_{L_{M}}$. The integer $M$ plays the role of a stability index. When the increments of the Brownian motion are too big, then a solution to $\bar{G}(x, \omega)=h_{0}$ may not exist.

Therefore $\bar{X}_{0}$ is an approximation of $X_{0}$, defined through (15)-(16) using an Euler approximation (with step size $\delta$ ) instead the flow $\Phi$ in $X=\Phi\left(X_{0}\right)$. In deterministic settings this is known as the shooting method to approximate ordinary differential equations with boundary conditions.

Now we study some stochastic differentiability properties of $X_{0}$ and $\bar{X}_{0}$. For this, let $\xi$ be a random variable such that $\bar{\Phi}_{1}\left(X_{0}\right)-\bar{\Phi}_{1}\left(\bar{X}_{0}\right)=\bar{\Phi}_{1}^{\prime}(\xi)\left(X_{0}-\bar{X}_{0}\right)$. Then, for $\omega \in L_{M}$, define $\bar{Z}(\xi)=F_{0}+F_{1} \bar{\Phi}_{1}^{\prime}(\xi)=\int_{0}^{1}\left(F_{0}+F_{1} \bar{\Phi}_{1}^{\prime}\left(\bar{X}_{0}+u\left(X_{0}-\bar{X}_{0}\right)\right) d u \neq 0\right.$. This gives

$$
X_{0}-\bar{X}_{0}=-(\bar{Z}(\xi))^{-1} F_{1}\left(\Phi_{1}\left(X_{0}\right)-\bar{\Phi}_{1}\left(X_{0}\right)\right),
$$

if $\omega \in L_{M}$. For $\omega \notin L_{M}$ we define $\bar{Z}(\xi)=1$. We will need the following stochastic differentiability properties of $X_{0}$ and $\bar{X}_{0}$.

Lemma 4.1. We have that $X_{0} \in \mathbb{D}^{\infty}, \bar{X}_{0} \in \mathbb{D}_{\text {loc }}^{\infty},(\bar{Z}(\xi))^{-1} \in \mathbb{D}_{\text {loc }}^{\infty}$. Also, for all $p>1, k=0,1, \ldots$,

$$
\begin{aligned}
& \sup _{\delta} \sup _{\left(t_{1}, \ldots, t_{k}\right) \in[0,1]^{k}}\left\|D_{t_{1}} \ldots D_{t_{k}} X_{0}\right\|_{p}+\sup _{\delta} \sup _{\left(t_{1}, \ldots, t_{k}\right) \in[0,1]^{k}}\left\|D_{t_{1}} \ldots D_{t_{k}} \bar{X}_{0}\right\|_{p} \\
& \quad+\sup _{\delta} \sup _{\left(t_{1}, \ldots, t_{k}\right) \in[0,1]^{k}}\left\|D_{t_{1}} \ldots D_{t_{k}}(\bar{Z}(\xi))^{-1}\right\|_{p}<\infty .
\end{aligned}
$$


Proof. In Kohatsu-Higa and Sanz-Solé [19] it is proven that $X_{0} \in \mathbb{D}^{\infty}$ and that

$$
D_{t} X_{0}=-\frac{F_{1} D_{t} \Phi_{1}\left(X_{0}\right)}{F_{0}+F_{1} \Phi_{1}^{\prime}\left(X_{0}\right)}
$$

Given that $F_{0} F_{1}>0$, we have $\left|D_{t} X_{0}\right| \leq C\left|D_{t} \Phi_{1}\left(X_{0}\right)\right|$. Therefore we obtain $\sup _{t}\left\|D_{t} X_{0}\right\|_{p}<\infty$ by applying Lemma 7.3. The proof of $\sup _{u, t}\left\|D_{u} D_{t} X_{0}\right\|_{p}<\infty$ is similar.

Now we will prove the properties about $\overline{X_{0}}$ and $(\bar{Z}(\xi))^{-1}$ for $k=1$.

We use the following localizing sequence: The sets

$$
L_{M}^{\epsilon}:=\left\{\max _{0 \leq k \leq N-1}\left|\omega\left(\Delta_{k}\right)\right|<M-2 \epsilon \text { or } \max _{0 \leq k \leq N-1}\left|\omega\left(\Delta_{k}\right)\right|>M+\epsilon\right\} \uparrow \Omega \text { as } \epsilon \rightarrow 0,
$$

and let $h_{M, \epsilon}: \mathbb{R}^{N} \rightarrow[0,1]$ be a bounded smooth function with bounded derivatives such that

$$
h_{M, \epsilon}\left(x_{1}, \ldots, x_{N}\right)= \begin{cases}1, & \text { if } \max \left\{\left|x_{1}\right|, \ldots,\left|x_{N}\right|\right\} \leq M-2 \epsilon, \\ 0, & \text { if } \max \left\{\left|x_{1}\right|, \ldots,\left|x_{N}\right|\right\} \geq M-\epsilon .\end{cases}
$$

Then the localizing random variable is defined by

$$
\overline{X_{0}}{ }^{\epsilon}=\overline{X_{0}} h_{M, \epsilon}\left(\omega\left(\Delta_{0}\right), \ldots, \omega\left(\Delta_{N-1}\right)\right)
$$

and

$\left(\bar{Z}(\xi)^{-1}\right)^{\epsilon}=(\bar{Z}(\xi))^{-1} h_{M, \epsilon}\left(\omega\left(\Delta_{0}\right), \ldots, \omega\left(\Delta_{N-1}\right)\right)+\left(1-h_{M, \epsilon}\left(\omega\left(\Delta_{0}\right), \ldots, \omega\left(\Delta_{N-1}\right)\right)\right)$.

Now, in order to prove the differentiability of ${\overline{X_{0}}}^{\epsilon}$ and $\left(\bar{Z}(\xi)^{-1}\right)^{\epsilon}$, consider $\omega^{n}=$ $\omega+n^{-1} \int_{0}^{*} g_{s} d s$ for $g \in L^{2}([0,1]), n \in \mathbb{N}$. To simplify notation we will write $h_{M, \epsilon}(\omega)$ for $h_{M, \epsilon}\left(\omega\left(\Delta_{0}\right), \ldots, \omega\left(\Delta_{N-1}\right)\right)$. Then consider

$$
\begin{aligned}
n\left(\overline{X_{0}}{ }^{\epsilon}\right. & \left.\left(\omega^{n}\right)-\overline{X_{0}}{ }^{\epsilon}(\omega)\right)-D_{g} \overline{X_{0}}(\omega) h_{M, \epsilon}(\omega)-\overline{X_{0}}(\omega) D_{g} h_{M, \epsilon}(\omega) \\
= & n\left(\overline{X_{0}}\left(\omega^{n}\right)-\overline{X_{0}}(\omega)-n^{-1} D_{g} \overline{X_{0}}(\omega)\right) h_{M, \epsilon}\left(\omega^{n}\right) \\
& +D_{g} \overline{X_{0}}(\omega)\left(h_{M, \epsilon}\left(\omega^{n}\right)-h_{M, \epsilon}(\omega)\right) \\
& +n\left(h_{M, \epsilon}\left(\omega^{n}\right)-h_{M, \epsilon}(\omega)-n^{-1} D_{g} h_{M, \epsilon}(\omega)\right) \overline{X_{0}}(\omega) .
\end{aligned}
$$

Here,

$$
\begin{aligned}
D_{g} \overline{X_{0}}(\omega) & =-\frac{F_{1}\left(D_{g} \bar{\Phi}_{1}\right)\left(\overline{X_{0}}\right)(\omega)}{F_{0}+F_{1} \bar{\Phi}_{1}^{\prime}\left(\overline{X_{0}}\right)(\omega)} \mathbb{I}_{L_{M}}(\omega), \\
D_{g} h_{M, \epsilon}(\omega) & =\sum_{j=1}^{N} \frac{\partial h_{M, \epsilon}}{\partial x_{j}}\left(\omega\left(\Delta_{0}\right), \ldots, \omega\left(\Delta_{N-1}\right)\right) \int_{t_{j-1}}^{t_{j}} g(s) d s .
\end{aligned}
$$

If we take $n$ big enough so that $\sqrt{\delta}\|g\|_{2} / n<\epsilon / 4$ and suppose that $h_{M, \epsilon}\left(\omega^{n}\right) \neq 0$, then, using the definition of $h_{M, \epsilon}$, we have

$$
\max _{0 \leq k \leq N-1}\left|\omega\left(\Delta_{k}\right)\right| \leq \max _{0 \leq k \leq N-1}\left|\omega^{n}\left(\Delta_{k}\right)\right|+\frac{\sqrt{\delta}\|g\|_{2}}{n} \leq M-\frac{3 \epsilon}{4} .
$$

Therefore we only consider the case $\omega, \omega^{n} \in L_{M}$. In such a case, both $\overline{X_{0}}(\omega)$ and $\overline{X_{0}}\left(\omega^{n}\right)$ satisfy the boundary condition. Subtracting these boundary conditions, we obtain

$$
n\left(\bar{X}_{0}\left(\omega^{n}\right)-\bar{X}_{0}(\omega)\right)=-F_{1} n\left(\frac{\bar{\Phi}_{1}\left(\omega^{n}, \bar{X}_{0}(\omega)\right)-\bar{\Phi}_{1}\left(\omega, \bar{X}_{0}(\omega)\right)}{F_{0}+F_{1} \bar{\Phi}_{1}^{\prime}\left(\omega^{n}, \xi^{n}(\omega)\right)}\right)
$$


where $\xi^{n}(\omega)$ is a random point between $\bar{X}_{0}(\omega)$ and $\bar{X}_{0}\left(\omega^{n}\right)$. Therefore, due to the stochastic differentiability of $\bar{\Phi}_{1}$ and the fact that $\xi^{n}(\omega)$ converges to $\bar{X}_{0}(\omega)$ in $L^{p}(\Omega)$ for any $p>1$ as $n \rightarrow \infty$, and using Lemma 7.3, it follows that

$$
E\left|\left(F_{1} n\left(\frac{\bar{\Phi}_{1}\left(\omega^{n}, \bar{X}_{0}(\omega)\right)-\bar{\Phi}_{1}\left(\omega, \bar{X}_{0}(\omega)\right)}{F_{0}+F_{1} \bar{\Phi}_{1}^{\prime}\left(\omega^{n}, \xi^{n}(\omega)\right)}\right)-\frac{F_{1}\left(D_{g} \bar{\Phi}_{1}\right)\left(\overline{X_{0}}\right)}{F_{0}+F_{1} \bar{\Phi}_{1}^{\prime}\left(\overline{X_{0}}\right)}\right) h_{M, \epsilon}\left(\omega^{n}\right)\right|^{p} \rightarrow 0 .
$$

The other terms in (24) are dealt in a similar fashion. The property that $\sup _{t, \delta}\left\|D_{t} \bar{X}_{0}\right\|_{p}<\infty$ is obtained through (25) using Lemma 7.3 and properties of the flow defined by $D_{t} \bar{\Phi}_{s}(x)$.

The proof of the stochastic differentiability of $\left(\bar{Z}(\xi)^{-1}\right)^{\epsilon}$ uses the same techniques. In fact, consider

$$
\begin{aligned}
& n\left(\left(\bar{Z}(\xi)^{-1}\right)\left(\omega^{n}\right)-\left(\bar{Z}(\xi)^{-1}\right)(\omega)\right) h_{M, \epsilon}\left(\omega^{n}\right)=h_{M, \epsilon}\left(\omega^{n}\right) F_{1} \\
& \times \frac{\int_{0}^{1}\left(\bar{\Phi}_{1}^{\prime}\left(\omega^{n},\left[\overline{X_{0}}+u\left(X_{0}-\overline{X_{0}}\right)\right]\left(\omega^{n}\right)\right)-\bar{\Phi}_{1}^{\prime}\left(\omega,\left[\overline{X_{0}}+u\left(X_{0}-\overline{X_{0}}\right)\right](\omega)\right) d u\right.}{\left(F_{0}+F_{1} \int_{0}^{1}\left(\bar{\Phi}_{1}^{\prime}\left(\omega^{n},\left[\overline{X_{0}}+u\left(X_{0}-\overline{X_{0}}\right)\right]\left(\omega^{n}\right)\right) d u\right)\left(F_{0}+F_{1} \int_{0}^{1}\left(\bar{\Phi}_{1}^{\prime}\left(\omega,\left[\overline{X_{0}}+u\left(X_{0}-\overline{X_{0}}\right)\right](\omega)\right) d u\right)\right.\right.} .
\end{aligned}
$$

Here, again $\overline{X_{0}}\left(\omega^{n}\right)$ and $\overline{X_{0}}(\omega)$ satisfy the boundary condition if $h_{M, \epsilon}\left(\omega^{n}\right) \neq$ 0 . Therefore the differentiability of $\left(\bar{Z}(\xi)^{-1}\right)^{\epsilon}$ follows from the differentiability properties of ${\overline{X_{0}}}^{\epsilon}$ and $\bar{\Phi}_{1}^{\prime}$.

Lemma 4.2. For $t \in[0,1]$ fixed, $k, p \in \mathbb{N}$ and for every $\epsilon \in(0,1)$ there exists a positive constant $C(\epsilon, k, p)$ such that

$$
\left\|X_{0}-\bar{X}_{0}^{\epsilon}\right\|_{k, p} \leq C(\epsilon, k, p) \delta^{1 / 2} .
$$

Proof. We have

$$
\begin{aligned}
X_{0}-\overline{X_{0}}{ }^{\epsilon} & =\left(X_{0}-\overline{X_{0}}\right) h_{M, \epsilon}+X_{0}\left(1-h_{M, \epsilon}\right) \\
& =(-\bar{Z}(\xi))^{-1} F_{1}\left(\Phi_{1}\left(X_{0}\right)-\bar{\Phi}_{1}\left(X_{0}\right)\right) h_{M, \epsilon}+X_{0}\left(1-h_{M, \epsilon}\right) .
\end{aligned}
$$

Lemma 4.1 and Proposition 3.1 give

$$
\left.\|(-\bar{Z}(\xi))^{-1} F_{1}\left(\Phi_{1}\left(X_{0}\right)-\bar{\Phi}_{1}\left(X_{0}\right)\right)\right) h_{M, \epsilon} \|_{k, p} \leq C(\epsilon, k, p) \delta^{1 / 2} .
$$

The second estimate $\left\|X_{0}\left(1-h_{M, \epsilon}\right)\right\|_{k, p} \leq C(\epsilon, k, p) \delta^{1 / 2}$ follows from (19).

Now we give the result that shows that the weak rate of convergence for our approximation method is $\delta$. Its proof will give us some important steps for the consideration of later approximations for the density of the solution process.

Theorem 4.2. Let $f \in C_{p}^{4}(\mathbb{R})$. Then we have that for any $t \in[0,1]$ there is a positive constant $C$, depending on $f$ but independent of $\pi, t$ and $\delta$, such that

$$
\left|E f\left(\Phi_{t}\left(X_{0}\right)\right)-E f\left(\bar{\Phi}_{t}\left(\bar{X}_{0}\right)\right)\right| \leq C \delta .
$$

The method of proof shown here has the advantage that it allows extension to the consideration of nonsmooth functions in cases where one has some estimations of the Malliavin covariance matrix of the process involved. In order to avoid a long proof with long expressions, we will sketch the proof of the above theorem using analogies with some of the steps taken in the proof of Theorem 3.1

Sketch of the proof of Theorem 4.2. First, Theorem3.1. is applicable due to Lemma 4.1, and therefore

$$
\left|E\left(f\left(\Phi_{t}\left(X_{0}\right)\right)-f\left(\bar{\Phi}_{t}\left(X_{0}\right)\right)\right)\right| \leq C \delta
$$


Now we consider the term

$$
\begin{aligned}
\left|E\left(f\left(\bar{\Phi}_{t}\left(X_{0}\right)\right)-f\left(\bar{\Phi}_{t}\left(\bar{X}_{0}\right)\right)\right)\right| \leq & \left|E\left(f\left(\bar{\Phi}_{t}\left(X_{0}\right)\right)-f\left(\bar{\Phi}_{t}\left(\bar{X}_{0}\right)\right) ; L_{M}\right)\right| \\
& +\left|E\left(f\left(\bar{\Phi}_{t}\left(X_{0}\right)\right)-f\left(\bar{\Phi}_{t}\left(\bar{X}_{0}\right)\right) ; \bar{L}_{M}\right)\right| .
\end{aligned}
$$

The second term on the right is smaller than $C \delta$ due to (19), Cauchy-Schwarz and the fact that $\sup _{\delta \in(0,1]} E\left|f\left(\bar{\Phi}_{t}\left(X_{0}\right)\right)-f\left(\bar{\Phi}_{t}\left(\bar{X}_{0}\right)\right)\right|^{p}<\infty$ for any $p$.

To deal with the first term, note that for $\omega \in L_{M}$, we have, using the mean value theorem and (20),

$$
\begin{aligned}
& f\left(\bar{\Phi}_{t}\left(X_{0}\right)-y\right)-f\left(\bar{\Phi}_{t}\left(\bar{X}_{0}\right)-y\right) \\
& \quad=\int_{0}^{1} \frac{d}{d x} f\left(\bar{\Phi}_{t}(\cdot-y)\right)\left(\bar{X}_{0}+u\left(X_{0}-\bar{X}_{0}\right)\right) d u\left(X_{0}-\bar{X}_{0}\right) \\
& \left.\quad=\int_{0}^{1} \frac{d}{d x} f\left(\bar{\Phi}_{t}(\cdot-y)\right)\left(\bar{X}_{0}+u\left(X_{0}-\bar{X}_{0}\right)\right) d u(-\bar{Z}(\xi))^{-1} F_{1}\left(\Phi_{1}\left(X_{0}\right)-\bar{\Phi}_{1}\left(X_{0}\right)\right)\right) .
\end{aligned}
$$

Therefore,

$$
\begin{aligned}
& E\left(f\left(\bar{\Phi}_{t}\left(\bar{X}_{0}\right)\right)-\right.\left.f\left(\bar{\Phi}_{t}\left(X_{0}\right)\right) ; L_{M}\right) \\
&=\sum_{i=1}^{N} E\left[\int_{0}^{1} \frac{d}{d x} f\left(\bar{\Phi}_{t}\left(\bar{X}_{0}+u\left(X_{0}-\bar{X}_{0}\right)\right)-y\right)\right) d u(-\bar{Z}(\xi))^{-1} F_{1} \\
& \cdot\left(\left.\frac{\partial}{\partial x} \Phi_{t}\left(t_{i}, x\right)\right|_{x=\bar{\Phi}_{t_{i-1}}\left(X_{0}\right)}\right. \\
& \times\left\{\bar{\Phi}_{t_{i}}\left(X_{0}\right)-\bar{\Phi}_{t_{i-1}}\left(X_{0}\right)-\left(\Phi_{t_{i}}\left(t_{i-1}, \bar{\Phi}_{t_{i-1}}\left(X_{0}\right)\right)-\bar{\Phi}_{t_{i-1}}\left(X_{0}\right)\right)\right\} \\
&\left.\left.+R_{i}\left(\bar{\Phi}_{t_{i}}\left(X_{0}\right)\right)-R_{i}\left(\Phi_{t_{i}}\left(t_{i-1}, \bar{\Phi}_{t_{i-1}}\left(X_{0}\right)\right)\right)\right) ; L_{M}\right]
\end{aligned}
$$

This is the analogue to formula (8). The residues $R_{i}$ are defined considering $f(x)=x$ in (9). As in Theorem 3.1, we arrive at the consideration of terms that are similar. For example, the analog to (III) is

$$
\begin{aligned}
& \int_{t_{i-1}}^{t_{i}} \int_{0}^{1} E\left(\frac { d } { d x } f \left(\overline { \Phi } _ { t } \left(\bar{X}_{0}+v\right.\right.\right.\left.\left.\left(X_{0}-\bar{X}_{0}\right)\right)-y\right)\left.(-\bar{Z}(\xi))^{-1} F_{1} \frac{\partial}{\partial x} \Phi_{t}\left(t_{i}, x\right)\right|_{x=\bar{\Phi}_{t_{i-1}}\left(X_{0}\right)} \\
&\left.\times\left.\int_{t_{i-1}}^{s} G\left(\Phi_{u}\left(t_{i-1}, \bar{\Phi}_{t_{i-1}}(x)\right)\right) d W_{u}\right|_{x=X_{0}} ; L_{M}\right) d v d s .
\end{aligned}
$$

Denote the expression inside the above expectation by $\Xi$. Then, for a fixed $\epsilon>0$,

$$
\begin{aligned}
& E\left(\Xi ; L_{M}\right)=E\left(\Xi h_{M, 2 \epsilon}\right)+E\left(\Xi\left(\mathbb{I}_{L_{M}}-h_{M, 2 \epsilon}\right)\right) \\
& \quad \leq E\left(\Xi^{\epsilon} h_{M, 2 \epsilon}\right)+C(\epsilon)\left(E\left(\Xi^{2}\right)\right)^{1 / 2} P\left(M-4 \epsilon \leq \sup _{0 \leq k \leq N-1}\left|W\left(\Delta_{k}\right)\right|<M\right)^{1 / 2},
\end{aligned}
$$

where $C(\epsilon)$ is a positive constant that depends only on $\epsilon$. $\Xi^{\epsilon}$ is the localization of $\Xi$ to the set $L_{M}^{\epsilon}$. That is, we put $\overline{X_{0}}=\overline{X_{0}}{ }^{\epsilon}$ and $\bar{Z}(\xi)^{-1}=\left(\bar{Z}(\xi)^{-1}\right)^{\epsilon}$ in the formula for $\Xi$.

Due to (19), we have that $P\left(M-4 \epsilon \leq \sup _{0 \leq k \leq N-1}\left|W\left(\Delta_{k}\right)\right|<M\right) \leq C(q) \delta^{\frac{q}{2}-1}$. 
For $E\left(\Xi^{\epsilon} h_{M, 2 \epsilon}\right)$ one can apply integration by parts. That is,

$$
\begin{aligned}
& E\left(\Xi^{\epsilon} h_{M, 2 \epsilon}\right) \\
& =E\left(\left.\frac{d}{d x} f\left(\bar{\Phi}_{t}\left(\bar{X}_{0}^{\epsilon}+v\left(X_{0}-\bar{X}_{0}^{\epsilon}\right)\right)-y\right)\left((-\bar{Z}(\xi))^{-1}\right)^{\epsilon} F_{1} \frac{\partial}{\partial x} \Phi_{t}\left(t_{i}, x\right)\right|_{x=\bar{\Phi}_{t_{i-1}}\left(X_{0}\right)}\right. \\
& \left.\quad \times\left.\int_{t_{i-1}}^{s} G\left(\Phi_{u}\left(t_{i-1}, \bar{\Phi}_{t_{i-1}}(x)\right)\right) d W_{u}\right|_{x=X_{0}} h_{M, 2 \epsilon}\right) .
\end{aligned}
$$

Now we can proceed, applying Lemma 7.5, to obtain that is enough to find a bound for a series of terms, one of which is

$$
\begin{aligned}
\sup _{u \in\left[t_{i-1}, t_{i}\right]} \mid \int_{0}^{1} E( & \left.D_{u} X_{0} f\left(\bar{\Phi}_{t}\left(\bar{X}_{0}+v\left(X_{0}-\bar{X}_{0}\right)\right)-y\right)\right) \\
\times(- & \bar{Z}(\xi))\left.^{-1} F_{1} \frac{\partial}{\partial x} \Phi_{t}\left(t_{i}, x\right)\right|_{x=\bar{\Phi}_{t_{i-1}}\left(X_{0}\right)} \\
& \left.\times \frac{\partial G\left(\Phi_{u}\left(t_{i-1}, \bar{\Phi}_{t_{i-1}}(\cdot)\right)\right)}{\partial x}\left(X_{0}\right) h_{M, 2 \epsilon}\right) .
\end{aligned}
$$

Given the bounds for all the processes involved, one has that the above expression is bounded by a constant that depends on $\epsilon$. For example, $\left|\left((-\bar{Z}(\xi))^{-1}\right)^{\epsilon}\right| \leq F_{0}^{-1}$ and $\left.f\left(\bar{\Phi}_{t}\left(\bar{X}_{0}^{\epsilon}+u\left(X_{0}-\bar{X}_{0}^{\epsilon}\right)\right)-y\right)\right) \in L^{p}(\Omega)$ for any $p>1, \epsilon>0$, uniformly in $u \in[0,1], \delta \in(0,1]$ and in the partition $\pi$.

Therefore the result follows.

Now we give the main result of this subsection:

Theorem 4.3. Suppose that $\sigma$ and $b$ are smooth functions with bounded derivatives, $F_{0} F_{1}>0$, and there exist $j_{0} \geq 0$ and $V \in \Sigma_{j_{0}}$ with $\left(V\left(X_{0}\right)\right)^{-1} \in \bigcap_{p \geq 1} L^{p}(\Omega)$. Then

$$
\sup _{y}\left|p(t, y)-E \phi_{\delta^{\frac{1}{2}}}\left(\bar{\Phi}_{t}\left(\bar{X}_{0}\right)-y\right)\right| \leq C \delta .
$$

Here $\phi_{r}$ denotes the density of a Gaussian random variable with mean 0 and standard deviation $r$, and $C$ is a positive constant independent of $\delta$ and the partition $\pi$.

Sketch of the proof of Theorem 4.3. First, one considers

$$
\begin{aligned}
p(t, y)-E \phi_{\delta^{\frac{1}{2}}}\left(\Phi_{t}\left(\bar{X}_{0}\right)-y\right)= & p(t, y)-E \phi_{\delta^{\frac{1}{2}}}\left(\Phi_{t}\left(X_{0}\right)-y\right) \\
& +E \phi_{\delta^{\frac{1}{2}}}\left(\Phi_{t}\left(X_{0}\right)-y\right)-E \phi_{\delta^{\frac{1}{2}}}\left(\bar{\Phi}_{t}\left(\bar{X}_{0}\right)-y\right) \\
:= & A+B .
\end{aligned}
$$

Now we prove that

$$
\sup _{y}|A| \leq C \delta
$$

Note that if $Z$ is a random variable with continuous density $q(z)$, then

$$
q(z)=\lim _{n \rightarrow \infty} E\left[\phi_{n^{-a}}(Z-z)\right] .
$$


Therefore,

$$
\begin{aligned}
A & =p(t, y)-E \phi_{\delta^{\frac{1}{2}}}\left(\Phi_{t}\left(X_{0}\right)-y\right) \\
& =\lim _{n \rightarrow \infty} E\left[\phi_{n^{-a}}\left(\Phi_{t}\left(X_{0}\right)-y\right)-\phi_{n^{-a}}\left(\Phi_{t}\left(X_{0}\right)+\delta^{\frac{1}{2}} \bar{W}_{1}-y\right)\right],
\end{aligned}
$$

where $n \in \mathbb{N}$ and $a>0$ is fixed. $\bar{W}$ is a Wiener process independent of $W$ and $E$ still denotes the expectation on the extended Wiener space supporting $(W, \bar{W})$. Here we apply a Taylor expansion argument

$$
\begin{gathered}
E\left[\phi_{n^{-a}}\left(\Phi_{t}\left(X_{0}\right)-y\right)-\phi_{n^{-a}}\left(\Phi_{t}\left(X_{0}\right)+\delta^{\frac{1}{2}} \bar{W}_{1}-y\right)\right]=E\left[\phi_{n^{-a}}^{\prime \prime}\left(\Phi_{t}\left(X_{0}\right)-y\right)\right] \frac{\delta}{2} \\
+\int_{0}^{1} E\left[\phi_{n^{-a}}^{\prime \prime \prime}\left(\Phi_{t}\left(X_{0}\right)-y+u \delta^{\frac{1}{2}} \bar{W}_{1}\right) \bar{W}_{1}^{3}\right] d u \frac{\delta^{\frac{3}{2}}}{3 !} .
\end{gathered}
$$

The proof of (28) is done if we prove that each of the expectations on the right hand side of (29) is bounded. Using Lemma 7.1, we have that for some universal constants $a, b, e, k$ and $w$

$$
\begin{aligned}
\left|E\left[\phi_{n^{-a}}^{\prime \prime}\left(\Phi_{t}\left(X_{0}\right)-y\right)\right]\right| & =\left|E\left[\Psi_{n^{-a}}\left(\Phi_{t}\left(X_{0}\right)-y\right) H^{3}\left(\Phi_{t}\left(X_{0}\right), 1\right)\right]\right| \\
& \leq\left\|H^{3}\left(\Phi_{t}\left(X_{0}\right), 1\right)\right\|_{1} \leq C\left\|\Delta_{\Phi_{t}\left(X_{0}\right)}^{-1}\right\|\left\|_{a}^{k}\right\| \Phi_{t}\left(X_{0}\right) \|_{e, b}^{w} .
\end{aligned}
$$

$\Psi_{r}$ denotes the distribution function of a $N(0, r)$ random variable. The right hand side of the above equation is bounded due to Theorem 4.1 and Proposition 3.1. Analogously we obtain

$$
\sup _{n} \sup _{u \in[0,1]} \sup _{\delta \in(0,1]} E\left[\phi_{n^{-a}}^{\prime \prime \prime}\left(\Phi_{t}\left(X_{0}\right)-y+u \delta^{\frac{1}{2}} \bar{W}_{1}\right) \bar{W}_{1}^{3}\right]<\infty .
$$

This finishes the proof of (28).

Now we proceed to prove that $\sup _{y}|B| \leq C \delta$. Assume without loss of generality that $t \in \pi$, and let $n(t)$ denote the integer $j$ such that $t_{j}=t$. Consider

$$
\begin{aligned}
B:= & E\left[\phi_{\delta^{\frac{1}{2}}}\left(\Phi_{t}\left(X_{0}\right)-y\right)-\phi_{\delta^{\frac{1}{2}}}\left(\bar{\Phi}_{t}\left(\bar{X}_{0}\right)-y\right)\right] \\
= & E\left[\phi_{\delta^{\frac{1}{2}}}\left(\Phi_{t}\left(X_{0}\right)-y\right)-\phi_{\delta^{\frac{1}{2}}}\left(\bar{\Phi}_{t}\left(X_{0}\right)-y\right)\right] \\
& +E\left[\phi_{\delta^{\frac{1}{2}}}\left(\bar{\Phi}_{t}\left(X_{0}\right)-y\right)-\phi_{\delta^{\frac{1}{2}}}\left(\bar{\Phi}_{t}\left(\bar{X}_{0}\right)-y\right)\right] \\
=: & B_{1}+B_{2} .
\end{aligned}
$$

Let's start with $B_{1}$. Define $\varphi_{\delta}(x)=\phi_{\delta^{\frac{1}{2}} / \sqrt{2}}(x)$ and $Z=\frac{\delta^{\frac{1}{2}}}{\sqrt{2}} \bar{W}_{1}$ First, apply (8) for $f=\varphi_{\delta}$ to obtain

$$
\begin{aligned}
& E \phi_{\delta^{\frac{1}{2}}}\left(\Phi_{t}\left(X_{0}\right)-y\right)-E \phi_{\delta^{\frac{1}{2}}}\left(\bar{\Phi}_{t}\left(X_{0}\right)-y\right) \\
& =E\left(\varphi_{\delta}\left(\Phi_{t}\left(X_{0}\right)+Z-y\right)-\varphi_{\delta}\left(\bar{\Phi}_{t}\left(X_{0}\right)+Z-y\right)\right) \\
& =-\sum_{i=1}^{n(t)} E\left[\left.\frac{d}{d y} \varphi_{\delta}\left(\Phi_{t}\left(t_{i}, x\right)+Z-y\right) \frac{d}{d x} \Phi_{t}\left(t_{i}, x\right)\right|_{x=\bar{\Phi}_{t_{i-1}}\left(X_{0}\right)}\right. \\
& \quad \times\left\{\bar{\Phi}_{t_{i}}\left(X_{0}\right)-\bar{\Phi}_{t_{i-1}}\left(X_{0}\right)-\left(\Phi_{t_{i}}\left(t_{i-1}, \bar{\Phi}_{t_{i-1}}\left(X_{0}\right)\right)-\bar{\Phi}_{t_{i-1}}\left(X_{0}\right)\right)\right\} \\
& \left.+R_{i}\left(\bar{\Phi}_{t_{i}}\left(X_{0}\right)\right)-R_{i}\left(\Phi_{t_{i}}\left(t_{i-1}, \bar{\Phi}_{t_{i-1}}\left(X_{0}\right)\right)\right)\right],
\end{aligned}
$$


where

$$
\begin{aligned}
R_{i}(U)= & \int_{0}^{1} \int_{0}^{s_{2}} \frac{d^{2}}{d x^{2}} \varphi_{\delta}\left(\Phi_{t}\left(t_{i}, \cdot\right)+Z-y\right) \\
& \times\left(U+s_{1}\left(U-\bar{\Phi}_{t_{i-1}}\left(X_{0}\right)\right)\right)\left(U-\bar{\Phi}_{t_{i-1}}\left(X_{0}\right)\right)^{2} d s_{1} d s_{2}
\end{aligned}
$$

Here the analysis goes as in the proof of Theorem 3.1. That is, one finds the expansion of $\bar{\Phi}_{t_{i}}\left(X_{0}\right)-\bar{\Phi}_{t_{i-1}}\left(X_{0}\right)-\left(\Phi_{t_{i}}\left(t_{i-1}, \bar{\Phi}_{t_{i-1}}\left(X_{0}\right)\right)-\bar{\Phi}_{t_{i-1}}\left(X_{0}\right)\right)$ using the Itô-Taylor formula.

Now we proceed to find uniform bounds for the expectations of the integrands as in (13). The additional problem that appears in this case is that the derivatives of $\varphi_{\delta}$ start to appear. Here we apply the integration by parts formula enough times so that we recover the function $\Psi$, which is bounded by 1 .

That is, consider for example the term analogous to the one obtained in (13). In such a case we have to prove that there exists a positive constant $C$, independent of the partition $\pi$ and $\delta$, such that

$$
\begin{aligned}
\Gamma & =\sup _{u \in\left[t_{i-1}, t_{i}\right]} \mid E\left(D _ { u } X _ { 0 } \frac { \partial G ( \Phi _ { u } ( t _ { i - 1 } , \overline { \Phi } _ { t _ { i - 1 } } ( \cdot ) ) ) } { \partial x } ( X _ { 0 } ) \frac { d } { d y } \varphi _ { \delta } \left(\Phi_{t}\left(t_{i}, \bar{\Phi}_{t_{i-1}}\left(X_{0}\right)\right)\right.\right. \\
& \left.+Z-y)\left.\frac{d}{d x} \Phi_{t}\left(t_{i}, x\right)\right|_{x=\bar{\Phi}_{t_{i-1}}\left(X_{0}\right)}\right) \mid \\
& \leq C .
\end{aligned}
$$

To prove the above inequality we will use Proposition 7.1 which, applied to $\Gamma$, gives that there exists appropiate constants such that

$$
\begin{aligned}
\Gamma=\sup _{u \in\left[t_{i-1}, t_{i}\right]} \mid E & \left(\Psi_{\delta^{1 / 2} / \sqrt{2}}\left(\Phi_{t}\left(t_{i}, \bar{\Phi}_{t_{i-1}}\left(X_{0}\right)\right)+Z-y\right) H^{2}\right. \\
& \times\left(\Phi_{t}\left(t_{i}, \bar{\Phi}_{t_{i-1}}\left(X_{0}\right)\right)+Z, D_{u} X_{0} \frac{\partial G\left(\Phi_{u}\left(t_{i-1}, \bar{\Phi}_{t_{i-1}}(\cdot)\right)\right)}{\partial x}\right) \\
\left.\left.\times\left.\left(X_{0}\right) \frac{d}{d x} \Phi_{t}\left(t_{i}, x\right)\right|_{x=\bar{\Phi}_{t_{i-1}}\left(X_{0}\right)}\right)\right) \mid & \\
\leq \sup _{u \in\left[t_{i-1}, t_{i}\right]} \| H^{2}\left(\Phi_{t}\left(t_{i}, \bar{\Phi}_{t_{i-1}}\left(X_{0}\right)\right)+Z,\right. & \left.\times\left. D_{u} X_{0} \frac{\partial G\left(\Phi_{u}\left(t_{i-1}, \bar{\Phi}_{t_{i-1}}(\cdot)\right)\right)}{\partial x}\left(X_{0}\right) \frac{d}{d x} \Phi_{t}\left(t_{i}, x\right)\right|_{x=\bar{\Phi}_{t_{i-1}}\left(X_{0}\right)}\right) \|_{1} \\
\leq \sup _{u \in\left[t_{i-1}, t_{i}\right]} & \left\{\left\|\Delta_{\Phi_{t}\left(t_{i}, \bar{\Phi}_{t_{i-1}}\left(X_{0}\right)\right)+Z}\right\|\left\|_{k}^{a}\right\| \Phi_{t}\left(t_{i}, \bar{\Phi}_{t_{i-1}}\left(X_{0}\right)\right)+Z \|_{e, b}^{w}\right. \\
\times & \left.\left\|\left.D_{u} X_{0} \frac{\partial G\left(\Phi_{u}\left(t_{i-1}, \bar{\Phi}_{t_{i-1}}(\cdot)\right)\right)}{\partial x}\left(X_{0}\right) \frac{d}{d x} \Phi_{t}\left(t_{i}, x\right)\right|_{x=\bar{\Phi}_{t_{i-1}}\left(X_{0}\right)}\right\|_{e^{\prime}, b^{\prime}}\right\} .
\end{aligned}
$$

The proof of (30) is finished if we prove the following assertions: 
(i) $\sup _{t_{i-1}<t_{i} \leq t}\left\|\Phi_{t}\left(t_{i}, \bar{\Phi}_{t_{i-1}}\left(X_{0}\right)\right)+Z\right\|_{d, b}<\infty$, for any $d, b \in \mathbb{N}$.

(ii) $\sup _{t_{i-1}<t_{i} \leq t}\left\|\left(\Delta_{\Phi_{t}\left(t_{i}, \bar{\Phi}_{t_{i-1}}\left(X_{0}\right)\right)+Z}\right)^{-1}\right\|_{p}<\infty$, for all $p>1$.

(iii) For all $d, b \in \mathbb{N}$,

$$
\begin{aligned}
& \sup _{u \in[0, t] ; t_{i-1}<u<t_{i} \leq t}\left\|\left(\left.D_{u} X_{0} \frac{\partial}{\partial x} G\left(\Phi_{u}\left(t_{i-1}, \bar{\Phi}_{t_{i-1}}\left(X_{0}\right)\right)\right) \frac{d}{d x} \Phi_{t}\left(t_{i}, x\right)\right|_{x=\bar{\Phi}_{t_{i-1}}\left(X_{0}\right)}\right)\right\|_{d, b} \\
& \quad<\infty .
\end{aligned}
$$

(i) and (iii) follow from flow properties, (21), Lemma 7.3 and Lemma 4.1. (ii) follows from Lemma 7.2 in the Appendix.

Now consider $B_{2}$, the other term in $B$. We have

$$
B_{2}=E\left[\left(\varphi_{\delta}\left(\bar{\Phi}_{t}\left(X_{0}\right)+Z-y\right)-\varphi_{\delta}\left(\bar{\Phi}_{t}\left(\bar{X}_{0}\right)+Z-y\right)\right)\left(1_{L_{M}}+1_{\bar{L}_{M}}\right)\right]=B_{21}+B_{22}
$$

In the case of $B_{22}$, using (19), one has for fixed $q \geq 5$

$$
\begin{aligned}
B_{22} & =E\left[\varphi_{\delta}\left(\bar{\Phi}_{t}\left(X_{0}\right)+Z-y\right)-\varphi_{\delta}\left(\bar{\Phi}_{t}\left(\bar{X}_{0}\right)+Z-y\right) ; \bar{L}_{M}\right] \\
& \leq C \delta^{-1 / 2} P\left(\bar{L}_{M}\right) \leq C \delta^{-1 / 2} \frac{\delta^{\frac{q}{2}-1}}{M^{q}} \leq C \delta,
\end{aligned}
$$

where $C$ is a positive constant that depends only on $q$ and $M$. Now consider $B_{21}$. For $\omega \in L_{M}$, we have using (26)

$$
\begin{aligned}
\varphi_{\delta}\left(\bar{\Phi}_{t}\left(X_{0}\right)+Z-y\right)-\varphi_{\delta}\left(\bar{\Phi}_{t}\left(\bar{X}_{0}\right)+Z-y\right) \\
=\int_{0}^{1} \frac{d}{d x} \varphi_{\delta}\left(\bar{\Phi}_{t}\left(\bar{X}_{0}+u\left(X_{0}-\bar{X}_{0}\right)\right)+Z-y\right) d u \\
\quad \times(-\bar{Z}(\xi))^{-1} F_{1}\left(\Phi_{1}\left(X_{0}\right)-\bar{\Phi}_{1}\left(X_{0}\right)\right) .
\end{aligned}
$$

Therefore the problem of considering the rate of convergence of $B_{21}$ goes through applying (8) localized on the set $L_{M}$ for $\Phi_{1}\left(X_{0}\right)-\bar{\Phi}_{1}\left(X_{0}\right)$. That is, $f(x)=x$ and $t=1$ in (8). Then one continues in the proof of Theorem 4.2. For example, instead of (27) we will have, for $\epsilon \in(0,1)$ fixed,

$$
\begin{aligned}
& \sup _{u \in\left[t_{i-1}, t_{i}\right]} \mid \int_{0}^{1} E\left(D_{u} X_{0} \frac{d}{d x} \varphi_{\delta}\left(\bar{\Phi}_{t}\left(\bar{X}_{0}^{\epsilon}+v\left(X_{0}-\bar{X}_{0}^{\epsilon}\right)\right)+Z-y\right)\right)\left((-\bar{Z}(\xi))^{-1}\right)^{\epsilon} F_{1} \\
& \left.\times\left.\frac{\partial}{\partial x} \Phi_{t}\left(t_{i}, x\right)\right|_{x=\bar{\Phi}_{t_{i-1}}\left(X_{0}\right)} \frac{\partial G\left(\Phi_{u}\left(t_{i-1}, \bar{\Phi}_{t_{i-1}}(\cdot)\right)\right)}{\partial x}\left(X_{0}\right) h_{M, 2 \epsilon}\right) d v \mid<C(\epsilon) .
\end{aligned}
$$

Here one can apply integration by parts and obtain the necessary properties as we have done in (31) (in particular, Lemma 7.2). Therefore the result follows.

As in the proof of Theorem [3.1, one has to deal with the residues $R_{i}(U)$ using the integration by parts formula (5).

With the same techniques as in this proof one can obtain results for approximations of distribution functions. 
4.2. Weak approximation for general boundary conditions in the multidimensional case. We will now briefly indicate how to obtain a weak approximation result for the multidimensional sde with boundary condition considered in [25]. In this section we will consider the stochastic differential equation

$$
d X_{t}+F\left(X_{t}\right) d t=B d W_{t}, \quad t \in[0,1]
$$

with boundary condition

$$
h\left(X_{0}, X_{1}\right)=\bar{h},
$$

for $h: \mathbb{R}^{2 d} \rightarrow \mathbb{R}^{d}$. Here $\left\{X_{t}, t \in[0,1]\right\}$ is an $\mathbb{R}^{d}$-valued continuous stochastic process and $\left\{W_{t}, t \in[0,1]\right\}$ is a $\mathbb{R}^{k}$-valued Brownian motion with $k \geq d ; F: \mathbb{R}^{d} \rightarrow$ $\mathbb{R}^{d}$ takes the form

$$
F(x)=A x+B \widetilde{f}(x),
$$

where $A$ is a $d \times d$ matrix, $\tilde{f}: \mathbb{R}^{d} \rightarrow \mathbb{R}^{k}$ is measurable and locally bounded, and $B$ is a $d \times k$ matrix. In 25] a theorem on existence and uniqueness of solution for this kind of equation was established. More explicitly, let $\mathcal{C}_{0}\left([0,1] ; \mathbb{R}^{k}\right)$ be the set of continuous, $\mathbb{R}^{d}$-valued functions vanishing at 0 ; set

$$
\Lambda=\left\{\int_{0}^{1} e^{A t} B d \varphi(t) ; \varphi \in \mathcal{C}\left([0,1] ; \mathbb{R}^{k}\right)\right\},
$$

where the integrals are defined using integration by parts. Assume

(H1): For any $z \in \Lambda$ the equation $h\left(y, e^{-A}(y+z)\right)=\bar{h}$ has a unique solution $y=g(z)$.

In order to find the solution of (34), (35) we consider the linear equation

$$
d Y_{t}+A Y_{t} d t=B d W_{t}, \quad t \in[0,1]
$$

with boundary condition (35). This equation has a unique solution, given by

$$
Y_{t}=e^{-A t}\left[g\left(\int_{0}^{1} e^{A s} B d W_{s}\right)+\int_{0}^{t} e^{A s} B d W_{s}\right] .
$$

Let

$$
\Sigma=\left\{\xi \in \mathcal{C}\left([0,1] ; \mathbb{R}^{k}\right), \xi_{t}-\xi_{0}+\int_{0}^{t} A \xi_{s} d s \in \operatorname{Im} B, 0 \leq t \leq 1, h\left(\xi_{0}, \xi_{1}\right)=\bar{h}\right\}
$$

Then there exists a bijection $\psi: \mathcal{C}_{0}\left([0,1] ; \mathbb{R}^{k}\right) \rightarrow \Sigma$ such that $Y_{t}=(\psi(W))_{t}$. Finally we define the mapping $T: \mathcal{C}_{0}\left([0,1] ; \mathbb{R}^{k}\right) \rightarrow \mathcal{C}_{0}\left([0,1] ; \mathbb{R}^{k}\right)$ by

$$
T(\theta)=\theta+\int_{0}^{\cdot} \widetilde{f}\left((\psi(\theta))_{s}\right) d s
$$

Theorem 4.4 (Nualart and Pardoux, [25]). Assume $T$ is a bijection and (H1) holds. Then equation (34) with boundary condition (35) possesses a unique solution in $C\left([0, T] ; \mathbb{R}^{d}\right)$ given by

$$
X=\psi\left(T^{-1}(W)\right) .
$$


Momentarily assume that $T$ is a bijection; then one can give a different way of expressing the solution to (34), (35). For this let $\theta=T^{-1}(W), \xi_{t}=\int_{0}^{t} e^{A u} B d W_{u}$ and $\varphi_{t}=\int_{0}^{t} e^{A u} B d \theta_{u}$. Then,

$$
\begin{aligned}
X_{t} & =e^{-A t}\left[g\left(\int_{0}^{1} e^{A u} B d \theta_{u}\right)+\int_{0}^{t} e^{A u} B d \theta_{u}\right] \\
& =e^{-A t}\left[g\left(\varphi_{1}\right)+\varphi_{t}\right] .
\end{aligned}
$$

In [10, it was proved that $\varphi_{t}=\xi_{t}+u_{t}$, where

$$
u_{t}=-\int_{0}^{t} e^{A s} B \tilde{f}\left(e^{-A s}\left[g\left(\xi_{1}+u_{1}\right)+\left(\xi_{s}+u_{s}\right)\right]\right) d s .
$$

Fix $y \in \mathbb{R}$, and assume that

$$
u_{t}(y)=-\int_{0}^{t} e^{A s} B \tilde{f}\left(e^{-A s}\left[g\left(\xi_{1}+y\right)+\xi_{s}+u_{s}(y)\right]\right) d s
$$

has a unique solution. Moreover, suppose that the mapping $y \mapsto u_{1}(y)$ has a unique fixed point $Y$. Then, clearly

$$
\varphi_{t}=\xi_{t}+u_{t}(Y)
$$

Let $|M|$ denote the norm of a matrix $M$, that is, $|M|=\sup _{|x|=1}|M x|$. We need the following assumption:

(H2): $g, \widetilde{f} \in C^{1}\left(\mathbb{R}^{d}, \mathbb{R}^{d}\right)$ and

$$
K\left(L_{\tilde{f}}, L_{g}\right)=L_{g}\left[\exp \left[\left(e^{2|A|}-1\right) \frac{|B|}{2|A|} L_{\widetilde{f}}\right]-1\right]<1
$$

where $\widetilde{f}$ and $g$ are Lipschitz functions with Lipschitz constants $L_{\widetilde{f}}$ and $L_{g}$, respectively.

Under (H2) $T$ is a bijection, and therefore there is a unique solution to (34) and (35).

Here we will work with the same approximation scheme as introduced in [10]. That is, define $\bar{\xi}_{t}, \bar{u}_{t}(y)$ by

$$
\begin{aligned}
& \bar{\xi}_{t}=\int_{0}^{t} e^{A \eta(s)} B d W_{s}, \\
& \bar{u}_{t}(y)=-\int_{0}^{t} e^{A \eta(s)} B \tilde{f}\left(e^{-A \eta(s)}\left[g\left(\bar{\xi}_{1}+y\right)+\bar{\xi}_{\eta(s)}+\bar{u}_{\eta(s)}(y)\right]\right) d s .
\end{aligned}
$$

In Section 3 of [10] it is proven that under $(\mathrm{H} 2) y \mapsto \bar{u}_{1}(y)$ has a unique fixed point, say $\bar{Y}$. Let $\bar{\varphi}_{t}=\bar{\xi}_{t}+\bar{u}_{t}(\bar{Y})$ and

$$
\bar{X}_{t}=e^{-A t}\left[g\left(\bar{\varphi}_{1}\right)+\bar{\varphi}_{t}\right] .
$$

Now we introduce some preliminary lemmas.

Lemma 4.3. Assume (H2). Then $I-u_{1}^{\prime}(y)$ and $I-\bar{u}_{1}^{\prime}(y)$ are invertible matrices for all $y \in \mathbb{R}^{d}$ a.s. 
Proof. We will sketch the proof for $I-\bar{u}_{1}^{\prime}(y)$.

It is enough to prove that the maximum eigenvalue of $\bar{u}_{1}^{\prime}(y)$ is strictly smaller than 1.

For this is enough to prove that $\left|\bar{u}_{1}\left(y_{1}\right)-\bar{u}_{1}\left(y_{2}\right)\right| \leq K\left(L_{\tilde{f}}, L_{g}\right)\left|y_{1}-y_{2}\right|<\left|y_{1}-y_{2}\right|$. This is exactly (3.16) in [10].

The following result is an extension of Lemma $3.2 \mathrm{in} \mathrm{19}$. The proof is obtained through standard methods of calculation for stochastic derivatives.

Lemma 4.4. Assume (H2). Then

(i) $u_{t}(y), \bar{u}_{t}(y) \in \mathbb{D}^{1, \infty}$, for all $y \in \mathbb{R}^{d}$ and $t \in[0,1]$.

(ii) $Y, \bar{Y} \in \mathbb{D}^{1, \infty}$ with

$$
D_{s} Y=\left(I-u_{1}^{\prime}(Y)\right)^{-1}\left(D_{s} u_{t}\right)(Y) \quad D_{s} \bar{Y}=\left(I-\bar{u}_{1}^{\prime}(\bar{Y})\right)^{-1}\left(D_{s} \bar{u}_{t}\right)(\bar{Y}) .
$$

(iii) $u_{t}(Y), \bar{u}_{t}(\bar{Y}) \in \mathbb{D}^{1, \infty}$, and the chain rule is satisfied for both processes for all $t \in[0,1]$.

(iv) $\sup _{s} E\left[\sup _{t}\left|D_{s}\left(u_{t}(Y)\right)\right|^{p}\right]<\infty$ and $\sup _{s} E\left[\sup _{t}\left|D_{s}\left(\bar{u}_{t}(\bar{Y})\right)\right|^{p}\right]<\infty$ for all $p>1$.

The following result gives the rate of convergence for the weak approximation. Strong approximations where studied in [10].

Theorem 4.5. Assume (H1), (H2), and that $f, \widetilde{f}$ and $g$ are in $C_{p}^{2}$. Then there exists a positive constant $C$, independent of $\delta$ and $\pi$, such that

$$
\left|E\left(f\left(X_{t}\right)-f\left(\bar{X}_{t}\right)\right)\right| \leq C \delta .
$$

Proof. First, note that

$$
f\left(X_{t}\right)-f\left(\bar{X}_{t}\right)=\Upsilon_{t}^{1}\left[\Upsilon_{t}^{2}\left(\varphi_{1}-\bar{\varphi}_{1}\right)+\varphi_{t}-\bar{\varphi}_{t}\right]
$$

where

$$
\begin{aligned}
& \Upsilon_{t}^{1}=\int_{0}^{1} f^{\prime}\left(\bar{X}_{t}+\alpha\left(X_{t}-\bar{X}_{t}\right)\right) d \alpha e^{-A t} \\
& \Upsilon_{t}^{2}=\int_{0}^{1} g^{\prime}\left(\bar{\varphi}_{1}+\alpha\left(\varphi_{1}-\bar{\varphi}_{1}\right)\right) d \alpha .
\end{aligned}
$$

Due to Lemma 4.4, we have that $\Upsilon^{1}, \Upsilon^{2} \in \mathbb{D}^{1, \infty}$.

Therefore we consider $\varphi_{t}-\bar{\varphi}_{t}=\xi_{t}-\bar{\xi}_{t}+\left(u_{t}(Y)-\bar{u}_{t}(\bar{Y})\right)$. The difference $\xi_{t}-\bar{\xi}_{t}$ can obviously be written as an expression of order $\delta$. That is,

$$
\xi_{t}-\bar{\xi}_{t}=\int_{0}^{t}\left(e^{A s}-e^{A \eta(s)}\right) B d W_{s}
$$

where $\left|e^{A s}-e^{A \eta(s)}\right| \leq e^{2|A|} \delta$, and therefore $\sup _{t \in[0,1]}\left\|\xi_{t}-\bar{\xi}_{t}\right\|_{p} \leq C(p) \delta$ for a positive constant $C(p)$ independent of $\delta$ and $\pi$ but depending on $p>1$.

To simplify the notation, define

$$
k\left(x_{1}, \ldots, x_{5}\right)=-e^{A x_{1}} B \widetilde{f}\left(e^{-A x_{1}}\left[g\left(x_{2}+x_{3}\right)+x_{4}+x_{5}\right]\right) .
$$


Now consider

(40)

$$
\begin{aligned}
u_{t}(Y)- & \bar{u}_{t}(\bar{Y}) \\
= & \int_{0}^{t} k\left(s, \xi_{1}, Y, \xi_{s}, u_{s}(Y)\right)-k\left(\eta(s), \bar{\xi}_{1}, Y, \bar{\xi}_{\eta(s)}, u_{s}(Y)\right) d s \\
& +\int_{0}^{t} k\left(\eta(s), \bar{\xi}_{1}, Y, \bar{\xi}_{\eta(s)}, u_{s}(Y)\right)-k\left(\eta(s), \bar{\xi}_{1}, \bar{Y}, \bar{\xi}_{\eta(s)}, u_{s}(Y)\right) d s \\
& +\int_{0}^{t} k\left(\eta(s), \bar{\xi}_{1}, \bar{Y}, \bar{\xi}_{\eta(s)}, u_{s}(Y)\right)-k\left(\eta(s), \bar{\xi}_{1}, \bar{Y}, \bar{\xi}_{\eta(s)}, \bar{u}_{s}(\bar{Y})\right) d s \\
& +\int_{0}^{t} k\left(\eta(s), \bar{\xi}_{1}, \bar{Y}, \bar{\xi}_{\eta(s)}, \bar{u}_{s}(\bar{Y})\right)-k\left(\eta(s), \bar{\xi}_{1}, \bar{Y}, \bar{\xi}_{\eta(s)}, \bar{u}_{\eta(s)}(\bar{Y})\right) d s \\
= & I_{1}(t)+I_{2}(t)+I_{3}(t)+I_{4}(t) .
\end{aligned}
$$

Let's start with $I_{4}$. Consider

$$
\begin{aligned}
\left|I_{4}(t)\right| & \leq \int_{0}^{t} e^{2|A| \eta(s)} B L_{\tilde{f}}\left|\bar{u}_{s}(\bar{Y})-\bar{u}_{\eta(s)}(\bar{Y})\right| d s \\
& \leq \delta B L_{\tilde{f}} \int_{0}^{t} e^{2|A| \eta(s)}\left|k\left(\eta(s), \bar{\xi}_{1}, \bar{Y}, \bar{\xi}_{\eta(s)}, \bar{u}_{\eta(s)}(\bar{Y})\right)\right| d s .
\end{aligned}
$$

Therefore it follows from Lemma 7.6 in the Appendix that $\left\|\sup _{s \in[0, t]}\left|I_{4}(s)\right|\right\|_{p} \leq$ $C(p) \delta$ for a positive constant $C(p)$ independent of $\delta$ and $\pi$ but depending on $p>1$. Now consider $I_{1}$. We will divide it into 4 terms:

$$
\begin{aligned}
I_{1}(t)= & \int_{0}^{t} k\left(s, \xi_{1}, Y, \xi_{s}, u_{s}(Y)\right)-k\left(\eta(s), \xi_{1}, Y, \xi_{s}, u_{s}(Y)\right) d s \\
& +\int_{0}^{t} k\left(\eta(s), \xi_{1}, Y, \xi_{s}, u_{s}(Y)\right)-k\left(\eta(s), \bar{\xi}_{1}, Y, \xi_{s}, u_{s}(Y)\right) d s \\
& +\int_{0}^{t} k\left(\eta(s), \bar{\xi}_{1}, Y, \xi_{s}, u_{s}(Y)\right)-k\left(\eta(s), \bar{\xi}_{1}, Y, \bar{\xi}_{s}, u_{s}(Y)\right) d s \\
& +\int_{0}^{t} k\left(\eta(s), \bar{\xi}_{1}, Y, \bar{\xi}_{s}, u_{s}(Y)\right)-k\left(\eta(s), \bar{\xi}_{1}, Y, \bar{\xi}_{\eta(s)}, u_{s}(Y)\right) d s \\
= & I_{11}(t)+I_{12}(t)+I_{13}(t)+I_{14}(t) .
\end{aligned}
$$

As in the case of $I_{4}$ and using the hypotheses on $\tilde{f}$ and $g$, one obtains

$$
\left\|\sup _{s \in[0, t]}\left|I_{1 i}(s)\right|\right\|_{p} \leq C(p) \delta
$$

for $i=1,2,3 . I_{14}$ can be written as (using the mean value theorem)

$$
I_{14}(t)=\Upsilon_{s}^{3}\left(W_{s}-W_{\eta(s)}\right)
$$

where

$$
\begin{aligned}
\Upsilon_{s}^{3}=e^{A \eta(s)} B \int_{0}^{1} \tilde{f}^{\prime}\left(e ^ { - A \eta ( s ) } \left[g \left(\bar{\xi}_{1}\right.\right.\right. & +Y) \\
& \left.\left.+\bar{\xi}_{\eta(s)}+\alpha\left(\bar{\xi}_{s}-\bar{\xi}_{\eta(s)}\right)+u_{s}(Y)\right]\right) d \alpha e^{A \eta(s)}
\end{aligned}
$$

Note that due to Lemma 4.4 one has that $\Upsilon^{3} \in \mathbb{D}^{1, \infty}$ 
Now we analyze the term $I_{3}$. We have, by the mean value theorem,

$$
I_{3}(t)=-\int_{0}^{t} \gamma_{s}\left(u_{s}(Y)-\bar{u}_{s}(\bar{Y})\right) d s
$$

where

$$
\begin{aligned}
\gamma_{s}=e^{A \eta(s)} B \int_{0}^{1} \tilde{f}^{\prime}\left(e ^ { - A \eta ( s ) } \left[g \left(\bar{\xi}_{1}\right.\right.\right. & +\bar{Y})+\bar{\xi}_{\eta(s)} \\
& \left.\left.+u_{s}(Y)+\theta\left(\bar{u}_{s}(\bar{Y})-u_{s}(Y)\right)\right]\right) e^{-A \eta(s)} d \theta .
\end{aligned}
$$

Similar calculations are applied to $I_{2}$ to obtain

$$
I_{2}(t)=-\int_{0}^{t} \beta_{s}(Y-\bar{Y}) d s
$$

where

$$
\begin{gathered}
\beta_{s}=e^{A \eta(s)} B \int_{0}^{1} \tilde{f}^{\prime}\left(e^{-A \eta(s)}\left[g\left(\bar{\xi}_{1}+Y+\theta(\bar{Y}-Y)\right)+\bar{\xi}_{\eta(s)}+u_{s}(Y)\right]\right) \\
\times e^{-A \eta(s)} g^{\prime}\left(\bar{\xi}_{1}+Y+\theta(\bar{Y}-Y)\right) d \theta
\end{gathered}
$$

Summarizing these calculations, we find that we can write (40) as

$$
u_{t}(Y)-\bar{u}_{t}(\bar{Y})=\int_{0}^{t} \alpha_{s} d s+\int_{0}^{t} \beta_{s}(Y-\bar{Y}) d s+\int_{0}^{t} \gamma_{s}\left(u_{s}(Y)-\bar{u}_{s}(\bar{Y})\right) d s .
$$

This is a linear equation in $u_{t}(Y)-\bar{u}_{t}(\bar{Y})$, where $\alpha$ is defined so that

$$
\int_{0}^{t} \alpha_{s} d s=\left(I_{1}+I_{4}\right)(t)
$$

Using classical results (see Dunford and Schwartz [9], vol II, page 1282), we solve (42):

$$
u_{t}(Y)-\bar{u}_{t}(\bar{Y})=\sum_{j=0}^{\infty} \Psi^{j} G(t)
$$

where

$$
G(t)=\int_{0}^{t} \alpha_{s} d s+\int_{0}^{t} \beta_{s} d s(Y-\bar{Y}) \quad \text { and } \quad(\Psi Z)(t)=\int_{0}^{t} \gamma_{s} Z(s) d s .
$$

Here $\sup _{s \in[0,1]}\left|\gamma_{s}\right|<\infty$ a.e. (see the proof of Lemma 7.7 in the Appendix), and therefore the series above converge uniformly for $t$ in compact sets.

In order to solve for $Y-\bar{Y}$ in (43) for $t=1$ one proves by induction (see Lemma 7.7 in the Appendix) that for $j=1,2, \ldots$

$$
\left|\Psi^{j-1} B(1)\right| \leq \frac{\left(e^{2|A|}-1\right)^{j}}{j ! 2^{j}|A|^{j}}|B|^{j} L_{\widetilde{f}}^{j} L_{g} .
$$

Therefore by (37) one has that $\left|\sum_{j=0}^{\infty} \Psi^{j} B(1)\right|<1$, and so the inverse of $I-\sum_{j=0}^{\infty} \Psi^{j} B(1)$ (45) exists.

Replacing $t=1$ in (43), we have, using (37),

$$
Y-\bar{Y}=\left(I-\sum_{j=0}^{\infty} \Psi^{j} B(1)\right)^{-1} \sum_{j=0}^{\infty} \Psi^{j} A(1),
$$


where $A(t)=\int_{0}^{t} \alpha_{s} d s+I_{1}(t)+I_{4}(t)$ and $B(t)=\int_{0}^{t} \beta_{s} d s$.

Using (45) in (43), we also have

$$
u_{t}(Y)-\bar{u}_{t}(\bar{Y})=\sum_{j=0}^{\infty} \Psi^{j} A(t)+\sum_{j=0}^{\infty} \Psi^{j} B(t)\left(I-\sum_{j=0}^{\infty} \Psi^{j} B(1)\right)^{-1} \sum_{j=0}^{\infty} \Psi^{j} A(1) .
$$

Note that $\left\|\sup _{t \in[0,1]}\left|A(t)-I_{14}(t)\right|\right\|_{p} \leq C \delta$. Now if we put all these estimates together, we have:

$$
\begin{aligned}
E\left[f\left(X_{t}\right)-f\left(\bar{X}_{t}\right)\right]= & E\left[\Upsilon_{t}^{1}\left[\Upsilon_{t}^{2}\left(\xi_{1}-\bar{\xi}_{1}\right)+\xi_{t}-\bar{\xi}_{t}\right]\right] \\
& +E\left[\Upsilon_{t}^{1} \Upsilon_{t}^{2}(Y-\bar{Y})\right]+E\left[\Upsilon_{t}^{1}\left(u_{t}(Y)-\bar{u}_{t}(\bar{Y})\right)\right] \\
= & A_{1}+A_{2}+A_{3} .
\end{aligned}
$$

First, it is easy to see that $\left|A_{1}\right| \leq C \delta$, given that $\sup _{t \in[0,1]}\left\|\xi_{t}-\bar{\xi}_{t}\right\|_{p} \leq C \delta$ and $\Upsilon^{1}$, $\Upsilon^{2} \in \mathbb{D}^{1, \infty}$.

Next, as $A_{2}$ and $A_{3}$ are of the same nature we only consider $A_{3}$, leaving $A_{2}$ to the reader. Using (46) one has

$$
\begin{aligned}
A_{3}=E\left[\Upsilon _ { t } ^ { 1 } \left\{\sum_{j=0}^{\infty} \Psi^{j}\left(A-I_{14}\right)(t)\right.\right. \\
\left.\left.\quad+\sum_{j=0}^{\infty} \Psi^{j} B(t)\left(I-\sum_{j=0}^{\infty} \Psi^{j} B(1)\right)^{-1} \sum_{j=0}^{\infty} \Psi^{j}\left(A-I_{14}\right)(1)\right\}\right] \\
+E\left[\Upsilon _ { t } ^ { 1 } \left\{\sum_{j=0}^{\infty} \Psi^{j} I_{14}(t)\right.\right. \\
\left.\left.\quad+\sum_{j=0}^{\infty} \Psi^{j} B(t)\left(I-\sum_{j=0}^{\infty} \Psi^{j} B(1)\right)^{-1} \sum_{j=0}^{\infty} \Psi^{j} I_{14}(1)\right\}\right] \\
=A_{31}+A_{32}
\end{aligned}
$$

In Lemma 7.8 in the Appendix it is proven that $\left|A_{31}\right| \leq C \delta$. To prove $\left|A_{32}\right| \leq C \delta$ one needs to apply the integration by parts formula (5) because the rate is being carried by $\left(W_{s}-W_{\eta(s)}\right)$ in the definition of $I_{14}$ in (41). This is done in Lemma 7.9 in the Appendix. This finishes the proof.

Further refining this proof, one could consider the approximations for densities or distribution functions of solutions to (34). The hypothesis will require further smoothness of $\widetilde{f}$ and $g$. The study of existence and smoothness of densities for multimensional stochastic differential equations with boundary conditions was carried out in [19]. The statement in the case of density approximations is

Theorem 4.6. Assume (H1) and (H2). Also assume that $g$ and $\tilde{f}$ are elements of $C_{p}^{\infty}$ and that $g^{\prime}(x)$ (respectively $\left.I+g^{\prime}(x)\right)$ has an inverse for all $x \in \mathbb{R}^{d}$ and that its inverse has at most polynomial growth at infinity. Then, if $\operatorname{det} B B^{T} \neq 0$ one has

$$
\sup _{x}\left|p(t, x)-E \phi_{\delta^{1 / 2}}\left(\bar{X}_{t}-x\right)\right| \leq C \delta,
$$

for a positive constant $C$ independent of $\delta$ and the partition $\pi$ and for $t \in[0,1)$ (respectively $t \in(0,1])$. 


\section{An EXAMPLE}

In this section we will discuss informally an example of a stochastic equation with boundary conditions. There are various examples where the methods introduced here can be applied. These include the smoothing problem (see [30]), the problem of estimating a maximum a posteriori for trajectories of diffusions processes (see [32] and [33]), and the study of some classes of reciprocal processes (see 20]). These equations also appear in the asymptotic study of waves in random media (see [11] and [29]) and in the study of second order stochastic differential equations (see [26]). In general, most of the control systems where differential equations with boundary conditions appear will have an associated stochastic differential equation with boundary conditions when noise is introduced into it. Usually the boundary conditions appear from using a space variable instead of a time variable. We will briefly discuss one of the possible applications where this is exactly the case. A more detailed account will be given in a future publication.

As a simple example we will give a description of the equation considered in 29]. In that article they considered a transmission-reflection problem for a one dimensional equation in a random slab. The coefficients are assumed to randomly fluctuate in a small scale, therefore producing a limit equation which will be a linear stochastic differential equation with linear boundary conditions. This limit equation is obtained using techniques of diffusion approximation. To describe the situation, let $L>0$ be fixed. The one dimensional acoustic wave equation in the interval $[0, L]$ is

$$
\begin{aligned}
\rho(x) \frac{\partial u}{\partial t}+\frac{\partial p}{\partial x} & =0, \\
\frac{1}{K(x)} \frac{\partial p}{\partial t}+\frac{\partial u}{\partial x} & =0,
\end{aligned}
$$

with some boundary conditions which will be described later. Here $u(x, t)$ is the velocity, $p(x, t)$ is the pressure, $\rho(x)$ is the density and $K(x)$ is the bulk modulus. Then one considers that $\rho(x)=1+\beta\left(Z_{x}^{\epsilon}\right)$ for some smooth function taking values in $[-c, c]$ for $c<1$ and $Z_{x}^{\epsilon}$ a Markov processes with certain properties. One also assumes that $K \equiv 1$, which corresponds to the homogeneous case. Then one defines $A=u+p$ and $B=u-p$, called the right and left going wave respectively. Then boundary conditions are set. In general they are of the type

$$
H_{0}\left(\begin{array}{c}
A \\
B
\end{array}\right)(0, t)+H_{1}\left(\begin{array}{c}
A \\
B
\end{array}\right)(L, t)=V_{0}
$$

for appropiate matrices $H_{0}, H_{1}$ and $V_{0}$. These conditions can correspond to entering pulses at $x=0$ and at $x=L$. A combination of pulses in $x=0$ and $x=L$ can also be considered. As described in [29, the general problem is, given these boundary conditions, what can we say about the medium if we have small-scale inhomogeneities present? (these being represented by $Z_{x}^{\epsilon}$ ).

In order to transform the above problem into a stochastic differential equation with boundary conditions, one considers the Fourier transforms of $A$ and $B$, which are random and will depend in general on $\epsilon$. Under certain conditions the limit equation for the Fourier transforms is given by

$$
\frac{d}{d x}\left(\begin{array}{c}
\tilde{A}^{\epsilon} \\
\tilde{B}^{\epsilon}
\end{array}\right)=\frac{i w}{2 \epsilon} \beta\left(Z_{x}^{\epsilon}\right)\left(\begin{array}{cc}
1 & e^{-2 i w x / \epsilon} \\
-e^{-2 i w x / \epsilon} & -1
\end{array}\right)\left(\begin{array}{c}
\tilde{A}^{\epsilon} \\
\tilde{B}^{\epsilon}
\end{array}\right),
$$


where $\tilde{A}^{\epsilon}(x, w)=A^{\epsilon}(x, w) e^{i w x / \epsilon}$ and $\tilde{B}^{\epsilon}(x, w)=B^{\epsilon}(x, w) e^{i w x / \epsilon}, A^{\epsilon}$ and $B^{\epsilon}$ being the Fourier transforms of $A$ and $B$. Some of the physical quantities of interest are $R=\tilde{B}^{\epsilon} / \tilde{A}^{\epsilon}$, the reflection coefficient, and $T=1 / \overline{\tilde{A}}^{\epsilon}$, the transmission coefficient. These quantities define one dimensional stochastic differential equations with boundary conditions.

The problems just described fall into a general category of diffusion approximation theorem from [13] which we quote here. In our case we will have $f=0, L=1$ and $\eta^{k}\left(t / \epsilon^{2}\right)=\beta\left(Z_{t}^{\epsilon}\right)$.

Theorem 5.1. Let $X^{\epsilon}$ be the smallest solution of the system

$$
\begin{aligned}
& \frac{d X_{t}^{\epsilon}}{d t}=f\left(t, X_{t}^{\epsilon}\right)+\sigma^{k}\left(t, X_{t}^{\epsilon}\right) \frac{1}{\epsilon} \eta^{k}\left(\frac{t}{\epsilon^{2}}\right), \\
& H_{0} X_{0}^{\epsilon}+H_{1} X_{1}^{\epsilon}=V_{0} .
\end{aligned}
$$

Here $\eta^{k}(t)$ are independent Markov processes with a unique invariant probability measure under which they are ergodic and fulfill Doeblin's condition. Denote $\alpha^{k}=$ $\int_{0}^{\infty} E\left[\eta^{k}(0) \eta^{k}(t)\right] d t \in(0, \infty)$. Let $X$ be the solution of

$$
\begin{aligned}
& d X_{t}=f\left(t, X_{t}\right) d t+\alpha^{k} \sigma^{k}\left(t, X_{t}\right) \circ d W_{t}^{k}, \\
& H_{0} X_{0}+H_{1} X_{1}=V_{0} .
\end{aligned}
$$

Then under conditions (H1) and (H2) (see 13]) there exists a unique solution $X$ in $\mathbb{L}_{C, \text { loc }}^{1,8}$, and furthermore $X^{\epsilon}$ converges weakly to $X$ in the uniform topology.

Therefore, depending on the properties of the process $\eta$, one will find a whole array of processes $X$ which satisfy equations (47) and (48), which according to our results can be approximated. As in the example considered, it is common that stochastic differential equations with boundary conditions appear when the variable $t$ represents a space variable rather than a time variable.

The conditions we have required in Theorem 4.3 are sufficient to obtain that (H1) and (H2) are satisfied. In the multidimensional case there are cases that we have considered here were (H2) is not satisfied.

\section{Conclusions}

We have considered an alternative method of proof for weak approximations of solutions of stochastic differential equations. This method should be useful in many cases, in particular, when nonadapted processes are considered or when we are interested in approximating the distribution or the density function of a locally smooth process.

Many variations of this argument can be implemented. For example, one can obtain with some further work an expansion of the error in terms of powers of the step size. One possible disadvantage of this method is that the calculation of the constants in this expansion is quite cumbersome. It does not seem to have a nice expression like in the case of diffusions (see e.g. [31]). Another problem of interest is to consider the generalization of Theorem 4.3 to many dimensions using the technique of stochastic invariant imbedding introduced in [13]. This extension covers a whole array of different applications in the area of second order stochastic differential equations. The techniques to cover this case should be similar to the one dimensional case expounded here. The added problem is that the results in [13] are local. In that case it is not straighforward to find a way to control the 
localization as has been done here through the set $L_{M}$. This topic, as well as some of the applications, will be covered in a future publication.

Another argument to approach the analysis of numerical schemes in nonlinear problems has been used to study the McKean-Vlasov equation (see [18]), and the author is currently considering applying a combination of both techniques to the case of the Burgers equation.

Further extensions of this method can be investigated in order to obtain weak approximation results for higher order schemes and to develop the error in powers of the step size of the approximation, which should help the design of interpolation schemes.

Also these ideas should shed some light on the behaviour of weak approximations to higher order sde's with boundary conditions, and also on stochastic partial differential equations with boundary conditions. These problems need to be studied further.

\section{Appendix}

In this section we will prove auxiliary results used in other sections.

The following proposition is the basic result to obtain properties of the densities of smooth random variables.

Proposition 7.1. Let $F=\left(F^{1}, \ldots, F^{d}\right) \in\left(\mathbb{D}^{\infty}\right)^{d}$ be such that

$$
\left(\operatorname{det} \Delta_{F}\right)^{-1} \in \bigcap_{p \geq 2} L^{p}(\Omega) \text {. }
$$

Let $G \in \mathbb{D}^{\infty}$ and $g \in C_{p}^{\infty}\left(\mathbb{R}^{d}\right)$. Then $\left(\operatorname{det} \Delta_{F}\right)^{-1} \in \mathbb{D}^{\infty}$, and for any multi-index $\alpha \in\{1, \ldots, d\}^{k}, k \geq 1$, there exists an element $H_{\alpha}(F, G) \in \mathbb{D}^{\infty}$ such that

$$
E\left[\left(\partial_{\alpha} g\right)(F) G\right]=E\left[g(F) H_{\alpha}(F, G)\right] .
$$

Furthermore for any multi-index $\alpha$ and any integers $p$ and $q$ there exist constants $C(p, q, \alpha), e, b, e^{\prime}, b^{\prime}, a, k$ and $w$ such that

$$
\left\|H_{\alpha}(F, G)\right\|_{p, q} \leq C(p, q, \alpha)\left\|\Delta_{F}^{-1}\right\|_{k}^{a}\|F\|_{e, b}^{w}\|G\|_{e^{\prime}, b^{\prime}} .
$$

Proof. The proof of this proposition can be obtained by performing some aditional calculations in the usual proof (see for example, Proposition 3.2.2 in Nualart 24]).

For example, let $\alpha=(1)$. Then it follows that

$$
H_{(1)}(F, G)=\sum_{j=1}^{d} \delta\left(G\left(\Delta_{F}^{-1}\right)^{1 j} D F^{j}\right) .
$$

Now, using the continuity of the adjoint operator $\delta$ and some standard properties of the norms $\|\cdot\|_{p, q}$, we have

$$
\begin{aligned}
\left\|\sum_{j=1}^{d} \delta\left(G\left(\Delta_{F}^{-1}\right)^{1 j} D F^{j}\right)\right\|_{p, q} & \leq\left\|\sum_{j=1}^{d} G\left(\Delta_{F}^{-1}\right)^{1 j} D F^{j}\right\|_{p+1, q} \\
& \leq C\left\|\Delta_{F}^{-1}\right\|_{p+1, b},\|F\|_{p+2, b^{\prime}}\|G\|_{p+1, b^{\prime \prime}}
\end{aligned}
$$


for some integers $b, b^{\prime}, b^{\prime \prime}$. To finish the proof is enough to note that

$$
D\left[\left(\Delta_{F}^{-1}\right)^{i j}\right]=-\sum_{k, l=1}^{d}\left(\Delta_{F}^{-1}\right)^{i k}\left(\Delta_{F}^{-1}\right)^{j l} D\left[\Delta_{F}^{k l}\right] .
$$

In the one dimensional case $(d=1)$ we write $H(F, G)=H_{(1)}(F, G)$. By induction we also define $H^{j}(F, G)=H^{j-1}(F, H(F, G))$.

Now we give some results on the estimation of Malliavin covariance matrices. In the following $F_{\delta}^{u}$ and $F^{u}$ denote measurable random fields.

Lemma 7.1. Let $F_{\delta}^{u}, F^{u} \in\left(\mathbb{D}^{1, \infty}\right)^{m}$ and suppose that the following conditons hold:

(i) There exists $\gamma>0$ such that $\sup \left\|F_{\delta}^{u}-F^{u}\right\|_{1, p}=O\left(\delta^{\gamma}\right)$, for all $p>1$.

(ii) $\sup _{u}\left\|\left(\operatorname{det} \Delta_{F^{u}}\right)^{-1}\right\|_{p}<\infty$, for all $p>1$.

(iii) For all $p$, there exists $\nu(p)>0$ such that $\sup _{u}\left\|\left(\operatorname{det} \Delta_{F_{\delta}^{u}}\right)^{-1}\right\|_{p}=O\left(\delta^{-\nu(p)}\right)$.

Then

$$
\sup _{\delta} \sup _{u}\left\|\left(\operatorname{det} \Delta_{F_{\delta}^{u}}\right)^{-1}\right\|_{p}<\infty \quad \text { for all } p>1 .
$$

Proof. Consider $A=\left[\left|\operatorname{det} \Delta_{F_{\delta}^{u}}-\operatorname{det} \Delta_{F^{u}}\right| \leq \frac{1}{2}\left|\operatorname{det} \Delta_{F^{u}}\right|\right]$. Then,

$$
\begin{gathered}
E\left(\left|\left(\operatorname{det} \Delta_{F_{\delta}^{u}}\right)^{-1}\right|^{p} ; A\right) \leq 2^{p} E\left(\left|\left(\operatorname{det} \Delta_{F^{u}}\right)^{-1}\right|^{p}\right)<\infty, \\
E\left(\left|\left(\operatorname{det} \Delta_{F_{\delta}^{u}}\right)^{-1}\right|^{p} ; A^{c}\right) \leq E\left(\left|\left(\operatorname{det} \Delta_{F_{\delta}^{u}}\right)^{-1}\right|^{2 p}\right)^{1 / 2} P\left(A^{c}\right)^{1 / 2} .
\end{gathered}
$$

We finish the proof by noting that

$$
P\left(A^{c}\right) \leq 2^{k} E\left(\left|\left(\operatorname{det} \Delta_{F^{u}}\right)^{-1}\right|^{k}\left|\operatorname{det} \Delta_{F_{\delta}^{u}}-\operatorname{det} \Delta_{F^{u}}\right|^{k}\right)=O\left(\delta^{\gamma k}\right),
$$

for any $k$. Taking $k$ big enough, we obtain that (49) is bounded.

As an application of this lemma we consider:

Lemma 7.2. Let $X_{0}$ and $\bar{X}_{0}^{\epsilon}$ be as defined in Section 4.1. Also let $Z=\frac{\delta^{\frac{1}{2}}}{\sqrt{2}} \bar{W}_{1}$, where $\bar{W}$ is a Wiener process independent of $W$. Then, for fixed $t>0$,

(I) $\sup _{t_{i-1}<t_{i} \leq t}\left\|\left(\Delta_{\Phi_{t}\left(t_{i}, \bar{\Phi}_{t_{i-1}}\left(X_{0}\right)\right)+Z}\right)^{-1}\right\|_{p}<\infty$, for all $p>1$, and

(II) $\sup _{u \in[0,1]}\left\|\left(\Delta_{\bar{\Phi}_{t}\left(\bar{X}_{0}^{\epsilon}+u\left(X_{0}-\bar{X}_{0}^{\epsilon}\right)\right)+Z}\right)^{-1}\right\|_{p}<\infty$, for all $p>1$.

Proof. In both cases we only need to check that the conditions in Lemma 7.1 are satisfied. For $(i)$ we have, due to Proposition 3.1 and Lemma 4.2

$$
\begin{aligned}
\left\|\Phi_{t}\left(t_{i}, \bar{\Phi}_{t_{i-1}}\left(X_{0}\right)\right)+Z-\Phi_{t}\left(X_{0}\right)\right\|_{1, p} & \leq C \delta^{1 / 2} \\
\left\|\sup _{u \in[0,1]} \bar{\Phi}_{t}\left(\bar{X}_{0}^{\epsilon}+u\left(X_{0}-\bar{X}_{0}^{\epsilon}\right)\right)+Z-\Phi_{t}\left(X_{0}\right)\right\|_{1, p} & \leq C \delta^{1 / 2},
\end{aligned}
$$

where $C$ is a positive constant that does not depend on $\delta$ or the partition $\pi$. (ii) follows from Theorem 4.1 (iii) is similar in both cases, so we will only do the first:

$$
\begin{aligned}
\Delta_{\Phi_{t}\left(t_{i}, \bar{\Phi}_{t_{i-1}}\left(X_{0}\right)\right)+Z} & =\int_{0}^{1}\left|D_{s} \Phi_{t}\left(t_{i}, \bar{\Phi}_{t_{i-1}}\left(X_{0}\right)\right)\right|^{2}+\left|\bar{D}_{s} Z\right|^{2} d s \geq \int_{0}^{1}\left|\bar{D}_{s} Z\right|^{2} d s \\
& \geq \frac{\delta}{2}
\end{aligned}
$$

From here the proof follows. 
Lemma 7.3. Let $\left\{F_{t}(x), t \in[0,1]\right\}, x \in \mathbb{R}^{k}$, be a family of stochastic processes such that there exists $\alpha \geq 1$ with

$$
\sup _{t \in[0,1]} E\left(\sup _{|x| \leq 1}\left|F_{t}(\cdot, n x)\right|^{p}\right) \leq K(p) n^{\alpha p}
$$

for any $p \geq 1$, where $K$ is a constant depending on $p$ and $\alpha$. Let $Y: \Omega \rightarrow \mathbb{R}^{k}$ be a random vector belonging to $\cap_{p \geq 1} L^{p}(\Omega)$. Then

$$
\begin{gathered}
\sup _{t \in[0,1]} E\left(\left|F_{t}(Y)\right|^{p}\right) \leq 2 K(p r)^{1 / r}\left(1+E|Y|^{(\alpha p+1) s}\right)^{\frac{1}{s}}, \\
\text { for any } p \geq 1 \text { and } \frac{1}{r}+\frac{1}{s}=1 r, s>1 .
\end{gathered}
$$

This result is Lemma 2.1 in 19. The above bound can be obtained from the proof. As an application one has the following result.

Lemma 7.4. Suppose that $\sigma$ and $m$ have bounded derivatives up to order $k$. Also let $Y: \Omega \rightarrow \mathbb{R}$ be a random variable belonging to $\bigcap_{p \geq 1} L^{p}(\Omega)$. Then, for each $p \geq 2$, there exists a constant $C_{k, p}$ such that

$$
\begin{aligned}
& \sup _{s \leq 1} E \sup _{t \in[s, 1]}\left\|\nabla^{k} \Phi_{t}(s, Y)\right\|^{p} \leq C_{k, p}, \\
& \sup _{s \leq 1} E \sup _{t \in[s, 1]}\left\|\nabla^{k} \bar{\Phi}_{t}(s, Y)\right\|^{p} \leq C_{k, p}, \\
& \sup _{s \leq 1} E \sup _{t \in[s, 1]}\left\|\nabla^{k}\left(\Phi_{t}(s, Y)-\bar{\Phi}_{t}(s, Y)\right)\right\|^{p} \leq C_{k, p} \delta^{p / 2} .
\end{aligned}
$$

The proofs of the above statements are obtained as in the proof of Proposition 3.1. That is, one applies Lemma 7.3 after checking that the condition (50) is satisfied. This is done for every $k$ using the Faà di Bruno formula (see [12]).

In the next lemma we obtain a formula to evaluate the expectations of products of random variables with stochastic integrals. The main ingredient of the proof is the integration by parts formula of Proposition 7.1 .

Lemma 7.5. Let $Y$ be a random variable such that $Y \in \bigcap_{p \geq 2} L^{p}(\Omega)$ and $Y \in \mathbb{D}^{1,4}$. Also let $\left\{u_{t}(x), t \in[0,1]\right\}, x \in \mathbb{R}$, be a family of measurable adapted random fields such that $u_{t}(x)$ and $\partial^{j} u_{t} / \partial x^{j}$ satisfy (50) for $j=1,2$.

Then for $a, b \in[0,1]$ and $X_{0} \in \mathbb{D}^{1, \infty}$, the following formula holds:

$$
E\left(\left.\int_{a}^{b} u_{t}(x) d W_{t}\right|_{x=X_{0}} Y\right)=E\left(\int_{a}^{b} Y D_{t} X_{0} \frac{\partial u_{t}}{\partial x}\left(X_{0}\right)+D_{t} Y u_{t}\left(X_{0}\right) d t\right)
$$

Sketch of the proof. Let $\phi_{r}(x)$ denote the density of a normal random variable with standard deviation $r$. The proof is obtained through the justification of the following 
steps:

$$
E\left(\left.\int_{a}^{b} u_{t}(x) d W_{t}\right|_{x=X_{0}} Y\right)=\lim _{r \rightarrow 0} E\left(\int_{\mathbb{R}} \int_{a}^{b} u_{t}(x) d W_{t} \phi_{r}\left(x-X_{0}\right) Y d x\right)
$$

$$
\begin{aligned}
& =\lim _{r \rightarrow 0} \int_{\mathbb{R}} E\left(\int_{a}^{b} u_{t}(x) d W_{t} \phi_{r}\left(x-X_{0}\right) Y\right) d x \\
& =\lim _{r \rightarrow 0} \int_{\mathbb{R}} E\left(\int_{a}^{b}\left(-\phi_{r}^{\prime}\left(x-X_{0}\right) D_{t} X_{0} Y+\phi_{r}\left(x-X_{0}\right) D_{t} Y\right) u_{t}(x) d t\right) d x \\
& =\lim _{r \rightarrow 0} E\left(\int_{a}^{b} \int_{\mathbb{R}} \phi_{r}\left(x-X_{0}\right)\left(D_{t} X_{0} Y u_{t}^{\prime}(x)+D_{t} Y u_{t}(x)\right) d x d t\right) \\
& =E\left(\int_{a}^{b} Y D_{t} X_{0} \frac{\partial u_{t}}{\partial x}\left(X_{0}\right)+D_{t} Y u_{t}\left(X_{0}\right) d t\right) .
\end{aligned}
$$

First the term on the left hand side of (51) is well defined due to the hypothesis and the application of Lemma 3.2.2 in [23] and Lemma 7.3. (52) follows because $\int_{\mathbb{R}} \int_{a}^{b} u_{t}(x) d W_{t} \phi_{r}\left(x-X_{0}\right) Y d x$ converges pointwise to $\left.\int_{a}^{b} u_{t}(x) d W_{t}\right|_{x=X_{0}} Y$, and this sequence is uniformly integrable. That is, one uses the hypothesis and Lemma 7.3 to prove that

$$
\sup _{r} E\left(\int_{\mathbb{R}} \int_{a}^{b} u_{t}(x) d W_{t} \phi_{r}\left(x-X_{0}\right) Y d x\right)^{2}<\infty .
$$

To prove the above one follows the following steps:

$$
\begin{aligned}
& E\left(\int_{\mathbb{R}} \int_{a}^{b} u_{t}(x) d W_{t} \phi_{r}\left(x-X_{0}\right) Y d x\right)^{2} \\
& =\sum_{n=0}^{\infty} E\left(\mathbb{I}\left(n \leq\left|X_{0}\right|<n+1\right) Y^{2}\left(\int_{\mathbb{R}} \int_{a}^{b} u_{t}(x) d W_{t} \phi_{r}\left(x-X_{0}\right) d x\right)^{2}\right) \\
& \leq \sum_{n=0}^{\infty} E\left(\mathbb{I}\left(n \leq\left|X_{0}\right|<n+1\right) Y^{2}\right. \\
& \times\left(\int_{\left\{\left|x-X_{0}\right| \leq \frac{|x|}{2}\right\}} \sup _{|x| \leq 2(n+1)}\left|\int_{a}^{b} u_{t}(x) d W_{t}\right| \phi_{r}\left(x-X_{0}\right) d x\right. \\
& \left.\left.+\int_{\left\{\left|x-X_{0}\right|>\frac{|x|}{2}\right\}}\left|\int_{a}^{b} u_{t}(x) d W_{t}\right| \phi_{r}\left(\frac{x}{2}\right) d x\right)^{2}\right) \\
& \leq C\left(\left(E Y^{4}\right)^{1 / 2}\left(E\left|X_{0}\right|^{k}\right)^{1 / 4}\left(\sum_{n=0}^{\infty} \frac{1}{n^{k / 4}}\left(E \sup _{|x| \leq 2(n+1)}\left|\int_{a}^{b} u_{t}(x) d W_{t}\right|^{8}\right)^{1 / 4}\right)\right. \\
& \left.+\sum_{n=0}^{\infty} \frac{1}{n^{k / 4}}\left(\int_{\mathbb{R}} E\left|\int_{a}^{b} u_{t}(x) d W_{t}\right|^{8} \phi_{r}\left(\frac{x}{2}\right) d x\right)^{1 / 4}\right)
\end{aligned}
$$


for any $k>0$. From here, using the Sobolev embedding theorem and the hypothesis, one finds bounds for the above expectations. These bounds are polynomials in $n$. Therefore, taking $k$ big enough, the uniform integrability follows.

(53) follows by Fubini's theorem. (54) is a consequence of the integration by parts formula. The last two steps in the proof are a repetition of the arguments used for 52) and (53).

Lemma 7.6. Let $k:[0,1]^{2} \times\left(\mathbb{R}^{d}\right)^{4} \rightarrow \mathbb{R}^{d \times d}$ be defined by (39). Then the following properties are verified:

(i) The function $k$ belongs to $C_{p}^{2}\left([0,1]^{2} \times\left(\mathbb{R}^{d}\right)^{4}\right)$ and furthermore the following inequalities are satisfied

$$
\begin{aligned}
&\left|k\left(x, x_{2}, \ldots, x_{5}\right)-k\left(y, x_{2}, \ldots, x_{5}\right)\right| \\
& \leq e^{2|A|} B|x-y|\left\{\left|\widetilde{f}\left(e^{-A x}\left[g\left(x_{2}+x_{3}\right)+x_{4}+x_{5}\right]\right)\right|\right. \\
&\left.+L_{\tilde{f}}\left|g\left(x_{2}+x_{3}\right)+x_{4}+x_{5}\right|\right\} . \\
&\left|k\left(x_{1}, x, x_{3}, \ldots, x_{5}\right)-k\left(x_{1}, y, x_{3}, \ldots, x_{5}\right)\right| \leq e^{2|A|} B L_{\tilde{f}} L_{g}|x-y| . \\
&\left|k\left(x_{1}, \ldots, x, x_{5}\right)-k\left(x_{1}, \ldots, y, x_{5}\right)\right| \leq e^{2|A|} B L_{\tilde{f}}|x-y| .
\end{aligned}
$$

(ii)

$$
\begin{array}{r}
E \sup _{s \in[0, t]}\left|k\left(\eta(s), \bar{\xi}_{1}, \bar{Y}, \bar{\xi}_{\eta(s)}, \bar{u}_{\eta(s)}(\bar{Y})\right)\right|^{p} \leq C(p), \\
E \sup _{s \in[0, t]}\left|k\left(s, \xi_{1}, Y, \xi_{s}, u_{s}(Y)\right)\right|^{p} \leq C(p),
\end{array}
$$

for a positive constant $C(p)$ independent of $\delta$ and $\pi$

Lemma 7.7. Under the conditions of Theorem 4.5 we have, for $j=1,2, \ldots$,

$$
\left|\Psi^{j-1} B(t)\right| \leq \frac{\left(e^{2|A| t}-1\right)^{j}}{j ! 2^{j}|A|^{j}}|B|^{j} L_{\widetilde{f}}^{j} L_{g} .
$$

Proof. Note that

$$
\begin{aligned}
& \left|\gamma_{s}\right| \leq e^{2|A| \eta(s)} B L_{\tilde{f}}, \\
& \left|\beta_{s}\right| \leq e^{2|A| \eta(s)} B L_{\tilde{f}} L_{g} .
\end{aligned}
$$

Therefore for $j=1$ we have

$$
|B(t)| \leq \int_{0}^{t}\left|\beta_{s}\right| d s \leq \frac{\left(e^{2|A| t}-1\right) B L_{\tilde{f}} L_{g}}{2|A|} .
$$

Now assume that the assertion is true for $j$. Then

$$
\begin{aligned}
\left|\Psi^{j} B(t)\right| & \leq \int_{0}^{t}\left|\Psi^{j-1} B(s)\right|\left|\gamma_{s}\right| d s \\
& \leq|B|^{j} L_{\widetilde{f}}^{j} L_{g} \int_{0}^{t} \frac{\left(e^{2|A| s}-1\right)^{j}}{j ! 2^{j}|A|^{j}} e^{2|A| \eta(s)} B L_{\tilde{f}} d s \\
& \leq \frac{\left(|B| L_{\tilde{f}}^{j+1} L_{g}\right.}{j ! 2^{j}|A|^{j}} \int_{0}^{t}\left(e^{2|A| s}-1\right)^{j} e^{2|A| s} d s \\
& \leq \frac{\left(|B| L_{\tilde{f}}\right)^{j+1} L_{g}}{j+1 ! 2^{j+1}|A|^{j+1}} \cdot
\end{aligned}
$$


Lemma 7.8. For $A(t)=I_{1}(t)+I_{4}(t)$, we have

$\left|E\left[\Upsilon_{t}^{1} \sum_{j=0}^{\infty} \Psi^{j}\left(A-I_{14}\right)(t)+\sum_{j=0}^{\infty} \Psi^{j} B(t)\left(I-\sum_{j=0}^{\infty} \Psi^{j} B(1)\right)^{-1} \sum_{j=0}^{\infty} \Psi^{j}\left(A-I_{14}\right)(1)\right]\right| \leq C \delta$

for some positive constant $C$ independent of $\delta$ and the partition $\pi$.

Proof. Given that $\Upsilon_{t}^{1} \in L^{p}(\Omega)$ for any $p>1$, and $\left|\sum_{j=0}^{\infty} \Psi^{j} B(t)\right|<1$, it is enough to prove that

$$
\begin{aligned}
E\left(\sum_{j=0}^{\infty}\left|\Psi^{j}\left(A-I_{14}\right)(t)\right|\right)^{p} & \leq C(p) \delta^{p}, \\
E\left|\left(I-\sum_{j=0}^{\infty} \Psi^{j} B(1)\right)^{-1} \sum_{j=0}^{\infty} \Psi^{j}\left(A-I_{14}\right)(1)\right|^{p} & \leq C(p) \delta^{p} .
\end{aligned}
$$

To prove the first inequality one proves by induction that

$$
\left|\Psi^{j}\left(A-I_{14}\right)(t)\right| \leq \sup _{s \in[0, t]}\left|\left(A-I_{14}\right)(s)\right| \frac{\left(e^{2|A|}-1\right)^{j}}{j ! 2^{j}|A|^{j}}|B|^{j} L_{\widetilde{f}}^{j},
$$

for $j=0,1, \ldots$ From here the inequality follows because

$$
E\left(\sup _{s \in[0, t]}\left|\left(A-I_{14}\right)(s)\right|^{p}\right)=E\left(\sup _{s \in[0, t]}\left|I_{11}(t)+I_{12}(t)+I_{13}(t)+I_{4}(t)\right|^{p}\right) \leq C(p) \delta^{p} .
$$

The second inequality in (55) is analogous except one also needs

$$
\left(I-\sum_{j=0}^{\infty} \Psi^{j} B(1)\right)^{-1} \in L^{p}(\Omega) \quad \text { for any } p>1 .
$$

This follows from (44), as

$$
\left|\left(I-\sum_{j=0}^{\infty} \Psi^{j} B(1)\right)^{-1}\right| \leq \frac{1}{1-K\left(L_{\tilde{f}}, L_{g}\right)} .
$$

\section{Lemma 7.9.}

$$
\left|E\left[\Upsilon_{t}^{1} \sum_{j=0}^{\infty} \Psi^{j} I_{14}(t)+\sum_{j=0}^{\infty} \Psi^{j} B(t)\left(I-\sum_{j=0}^{\infty} \Psi^{j} B(1)\right)^{-1} \sum_{j=0}^{\infty} \Psi^{j} I_{14}(1)\right]\right| \leq C \delta,
$$

where $C$ is a positive constant that does not depend on $\delta$ or the partition $\pi$. 
Proof. Consider first, for $j \geq 1$, the term

(56)

$$
\begin{aligned}
E\left[\Upsilon_{t}^{1}\right. & \left.\Psi^{j} I_{14}(t)\right] \\
= & \int_{0}^{t} \int_{0}^{s_{j-1}} \cdots \int_{0}^{s_{2}} E\left[\prod_{l=1}^{j-1} \gamma_{s_{l}} \Upsilon_{t}^{1} \Upsilon_{s_{1}}^{3}\left(W_{s_{1}}-W_{\eta\left(s_{1}\right)}\right)\right] d s_{1} \cdots d s_{j-1} \\
= & \int_{0}^{t} \int_{0}^{s_{j-1}} \cdots \int_{0}^{s_{2}} \int_{\eta\left(s_{1}\right)}^{s_{1}} \\
& \times E\left[D_{u}\left(\Upsilon_{t}^{1} \Upsilon_{s_{1}}^{3}\right) \prod_{l=1}^{j-1} \gamma_{s_{l}}+\sum_{k=1}^{j-1} D_{u}\left(\gamma_{s_{k}}\right) \prod_{l=1, l \neq k}^{j-1} \gamma_{s_{l}} \Upsilon_{t}^{1} \Upsilon_{s_{1}}^{3}\right] d u d s_{1} \cdots d s_{j-1} \\
\leq & C\left(1+C_{1}(j-1)\right) \delta \frac{\left(e^{2|A|}-1\right)^{j-1}}{j-1 ! 2^{j-1}|A|^{j-1}}|B|^{j-1} L_{\tilde{f}}^{j-1} .
\end{aligned}
$$

The above calculation follows from (5) and previous estimates on $\Upsilon^{1}, \Upsilon^{3}$ and $\gamma$. Also note that a calculation gives

$$
\sup _{u \leq s}\left\|D_{u} \gamma_{s}\right\|_{p} \leq C(p) e^{2|A| \eta(s)} B .
$$

A calculation for the other terms is done noting that we have to prove some differentiability properties of $\sum_{j=0}^{\infty} \Psi^{j} B(t)\left(I-\sum_{j=0}^{\infty} \Psi^{j} B(1)\right)^{-1}$. Given that $\beta_{s} \in$ $\mathbb{D}^{1, \infty}$ uniformly in $s \in[0,1]$, one can obtain the same property for $\sum_{j=0}^{\infty} \Psi^{j} B(t)$, as we have shown in (56). Similarly for $\left(I-\sum_{j=0}^{\infty} \Psi^{j} B(1)\right)^{-1}$, if one uses the inversion formula $(I-M)^{-1}=\sum_{j=0}^{\infty} M^{j}$ for a matrix $M$ with norm smaller than 1.

\section{REFERENCES}

[1] M.B. Adams, A.S. Willsky and B.C. Levy, Linear estimation of boundary value stochastic processes, IEEE Trans. Automat. Control AC- 29 (1984) 803-821. MR 85k:93064 MR 86a:93135

[2] H. Ahn and A. Kohatsu-Higa, The Euler scheme for anticipating stochastic differential equations, Stochastics and Stochastics Rep. 54 (1995) 247-269. MR 97b:60098

[3] V. Bally and D. Talay, The law of the Euler scheme for stochastic differential equations I: convergence rate of the distribution function, Probab. Theory Related Fields 104 (1996) 43-60. MR 96k:60136

[4] V. Bally and D. Talay, The law of the Euler scheme for stochastic differential equations: II. Convergence rate of the density, Monte Carlo Methods Appl. 2 (1996) 93-128. MR 97k:60157

[5] M. Bossy and D. Talay, A Stochastic Particle Method for the McKean-Vlasov and Burgers equations, Math. Comp. 66 (1997) 157-192. MR 97c:60233

[6] M. Bossy and D. Talay, Convergence rate for the approximation of the limit law of weakly interacting particles: application to the Burgers equation, Ann. Appl. Probab. 6 (1996) 818-861. MR 97k:60158

[7] R. Bellman and G.M. Wing, An introduction to invariant imbedding, Wiley, New York, 1976. MR 52:12558

[8] C. Donati-Martin, Equations différentielles stochastiques dans $\mathbb{R}$ avec conditions aux bords, Stochastics Stochastics Rep. 35 (1991) 143-173. MR 92h:60091

[9] N. Dunford and L. Schwartz, Linear Operators, Part II, Pure and Applied Mathematics, A Series of Texts and Monographs, Intescience Pub., 1964. MR 32:6181

[10] M. Ferrante, A. Kohatsu-Higa and M. Sanz-Solé, Strong approximations for stochastic differential equations with boundary conditions, Stochastic Processes Appl. 61 (1996) 323-337. MR 97b:60099 
[11] J.P. Fouque and E. Merzbach, A limit theorem for linear boundary value problems in random media, Ann. Appl. Probab. 4 (1994) 549-569. MR 95e:60063

[12] L. E. Fraenkel, Formulae for high derivatives of composite functions, Math. Proc. Cambridge Philos. Soc. 83 (1978) 159-165. MR 58:6124

[13] J. Garnier, Stochastic invariant imbedding. Application to stochastic differential equations with boundary conditions, Probab. Theory Related Fields 102 (1995) 249-271. MR 96k:60138

[14] Y. Hu and S. Watanabe, Donsker delta functions and approximations of heat kernels by the time discretization method, J. Math. Kyoto Univ. 36 (1996) 499-518. MR 97k:60154

[15] H. Keller, Numerical Methods for Two-Point Boundary-Valued Problems, Blaisdell, 1968. MR 37:6038

[16] P.E. Kloeden and E. Platen, Numerical Solution of Stochastic Differential Equations, Applications of Mathematics 23, Springer, New York, 1992. MR 94b:60069

[17] A. Kohatsu-Higa, High Itô-Taylor approximations to heat kernels, J. Math. Kyoto Univ. 37 (1997) 129-150 (1997). MR 98c:60060

[18] A. Kohatsu-Higa and S. Ogawa, Weak rate of convergence for an Euler scheme of nonlinear SDE's, Monte Carlo Methods Appl. 3 (1997) 327-345. MR 98i:60053

[19] A. Kohatsu-Higa and M. Sanz-Solé, Existence and regularity of the density for solutions to stochastic differential equations with boundary conditions, Stochastics Stochastics Rep. 60 (1997) 1-22. MR 98d:60111

[20] A. Krener, Reciprocal processes and the stochastic realization problem for acausal system, Modelling, identification and robust control, C.I. Brynes and E. Lindquist, eds., Elsevier/North Holland, Amsterdam, New York, 1986, pp. 197-209. MR 89c:93065.

[21] H. Kwakernaak, Periodic linear differential stochastic processes, SIAM J. Control Optim. 13 (1975) 400-413. MR 51:15114

[22] G.N. Milshtein, A method of second order accuracy integration of stochastic differential equations Theor. Probab. Appl. 23 (1978) 396-401. MR 58:24549

[23] D. Nualart, The Malliavin Calculus and Related Topics, Springer Verlag, 1995. MR 96k:60130

[24] D. Nualart, Analysis on Wiener space and anticipating stochastic calculus, Lecture Notes in Mathematics vol. 1690, Ecole d'Été de Probabilités de Saint-Flour XXV, Springer, Berlin, 1998, 123-227. MR 99k:60144

[25] D. Nualart and E. Pardoux, Boundary value problems for stochastic differential equations, Ann. Probab. 19 (1991) 1118-1144. MR 92j:60072

[26] D. Nualart and E. Pardoux, Second order stochastic differential equations with Dirichlet boundary conditions, Stochastic Processes Appl. 39 (1991) 1-24. MR 93b:60123

[27] D. Ocone and E. Pardoux, Linear Stochastic Differential Equations with boundary conditions, Probab. Theory Related Fields 82 (1989) 489-526. MR 91a:60154

[28] D. Ocone and E. Pardoux, A generalized Itô-Ventzell formula. Application to a class of anticipating stochastic differential equations, Ann. Inst. H. Poincaré Probab. Statist. 25 (1989) 39-71. MR 90g:60055

[29] G. Papanicolaou, Waves in one dimensional random media, Lecture Notes in Mathematics vol. 1362, Ecole d'Été de Probabilités de Saint-Flour XXV (1985-87), Springer, Berlin, 1988, 205-276. MR 90j:82004

[30] E. Pardoux, Equations du lissage nonlineaire, Lect. Notes Control Inf. Sci. vol. 61, Filtering and Control of Random Processes, H. Korezlioglu, G. Mazziotto and J. Szpirglas, eds., Springer, Berlin, 1986, 206-218. MR 87i:93104

[31] D. Talay and L. Tubaro, Expansion of the global error for numerical schemes solving stochastic differential equations, Stochastic Anal. Appl. 8 (1990) 483-510. MR 92e:60124

[32] O. Zeitouni and A. Dembo, A maximum a posteriori estimator for trajectories of diffusions, Stochastics 20 (1987) 221-246 (Erratum) 20 (1987) 341. MR 88b:93050a,b

[33] O. Zeitouni and A. Dembo, An existence theorem for maximum a posteriori estimation of trajectories of diffusions, Stochastics 23 (1988) 197-218. MR 89g:93100

[34] O. Zeitouni and A. Dembo, A change of variables formula for Stratonovich integrals and existence of solutions for two-point stochastic boundary value problems, Probab. Theory Related Fields 84 (1990) 411-425. MR 91h:60061

Universitat Pompeu Fabra. Departament d’Economia. Ramón Trias Fargas 25-27. 08005 Barcelona. Spain

E-mail address: kohatsu@upf.es 